\title{
Boundary Value Problems for Systems of Second-Order Dynamic Equations on Time Scales with $\Delta$-Carathéodory Functions
}

\author{
M. Frigon ${ }^{1}$ and H. Gilbert ${ }^{2}$ \\ ${ }^{1}$ Département de Mathématiques et de Statistique, Université de Montréal, CP 6128, \\ Succursale Centre-Ville, Montréal, QC, Canada H3C 3J7 \\ ${ }^{2}$ Département de Mathématiques, College Édouard-Montpetit, 945 Chemin de Chambly, \\ Longueuil, QC, Canada J4H 3M6
}

Correspondence should be addressed to M. Frigon, frigon@dms.umontreal.ca

Received 31 August 2010; Accepted 30 November 2010

Academic Editor: J. Mawhin

Copyright (c) 2010 M. Frigon and H. Gilbert. This is an open access article distributed under the Creative Commons Attribution License, which permits unrestricted use, distribution, and reproduction in any medium, provided the original work is properly cited.

We establish the existence of solutions to systems of second-order dynamic equations on time scales with the right member $f$, a $\Delta$-Carathéodory function. First, we consider the case where the nonlinearity $f$ does not depend on the $\Delta$-derivative, $x^{\Delta}(t)$. We obtain existence results for Strum-Liouville and for periodic boundary conditions. Finally, we consider more general systems in which the nonlinearity $f$ depends on the $\Delta$-derivative and satisfies a linear growth condition with respect to $x^{\Delta}(t)$. Our existence results rely on notions of solution-tube that are introduced in this paper.

\section{Introduction}

In this paper, we establish existence results for the following systems of second-order dynamic equations on time scales:

$$
\begin{gathered}
x^{\Delta \Delta}(t)=f(t, x(\sigma(t))), \quad \Delta \text {-a.e. } t \in \mathbb{T}_{0}^{\kappa^{2}}, x \in(\mathrm{BC}), \\
x^{\Delta \Delta}(t)=f\left(t, x(\sigma(t)), x^{\Delta}(t)\right), \quad \Delta \text {-a.e. } t \in \mathbb{T}_{0}^{\kappa^{2}}, \\
a_{0} x(a)-x^{\Delta}(a)=x_{0}, \quad a_{1} x(b)+\gamma_{1} x^{\Delta}(\rho(b))=x_{1} .
\end{gathered}
$$


Here, $\mathbb{T}$ is a compact time scale where $a=\min \mathbb{T}, b=\max \mathbb{T}$, and $\mathbb{T}_{0}^{\kappa^{2}}$ is defined in (2.4). The map $f: \mathbb{T}_{0}^{\kappa} \times \mathbb{R}^{n} \rightarrow \mathbb{R}^{n}$ is $\Delta$-Carathéodory (see Definition 2.9), and (BC) denotes one of the following boundary conditions:

$$
\begin{gathered}
x(a)=x(b), \\
x^{\Delta}(a)=x^{\Delta}(\rho(b)), \\
a_{0} x(a)-\gamma_{0} x^{\Delta}(a)=x_{0}, \\
a_{1} x(b)+\gamma_{1} x^{\Delta}(\rho(b))=x_{1},
\end{gathered}
$$

where $a_{0}, a_{1}, \gamma_{0}, \gamma_{1} \geq 0, \max \left\{a_{0}, \gamma_{0}\right\}>0$, and $\max \left\{a_{1}, \gamma_{1}\right\}>0$.

Problem (1.1) was mainly treated in the case where it has only one equation $(n=1)$ and $f$ is continuous. In particular, the existence of a solution of (1.1) was established by Akın [1] for the Dirichlet boundary condition and by Stehlík [2] for the periodic boundary condition. Equation (1.1) with nonlinear boundary conditions was studied by Peterson et al. [3]. In all those results, the method of lower and upper solutions was used. See also [4, 5] and the references therein for other results on the problem (1.1) when $n=1$.

Very few existence results were obtained for the system (1.1) when $n>1$. Recently, Henderson et al. [6] and Amster et al. [7] established the existence of solutions of (1.1) with Sturm-Liouville and nonlinear boundary conditions, respectively, assuming that $f$ is a continuous function satisfying the following condition:

$$
\exists R>0 \text { such that }\langle x, f(t, x)\rangle>0 \quad \text { if }\|x\|=R \text {. }
$$

The fact that the right member in the system (1.2) depends also on the $\Delta$-derivative, $x^{\Delta}$, increases considerably the difficulty of this problem. So, it is not surprising that there are almost no results for this problem in the literature. Atici et al. [8] studied this problem in the particular case, where there is only one equation $(n=1)$ and $f$ is positive, continuous and satisfies a monotonicity condition. Assuming a growth condition of Wintner type and using the method of lower and upper solutions, they obtained the existence of a solution.

The system (1.2) with the Dirichlet boundary condition was studied by Henderson and Tisdell [9] in the general case where $n>1$. They considered $f$ a continuous function and $\mathbb{T}$ a regular time scale (i.e., $\rho(t)<t<\sigma(t)$ or $\mathbb{T}=[a, b]$ ). They established the existence of a solution of (1.2) under the following assumptions:

(A1) there exists $R>\max \left\{\left\|x_{0}\right\|,\left\|x_{1}\right\|\right\}$ such that $2\langle x, f(t, x, y)\rangle+\|y\|^{2}>0$ if $\|x\|=R$, $-2\langle x, y\rangle \leq \mu(t)\|y\|^{2}$,

(A2) there exist $c, d \geq 0$ such that $d(\rho(b)-a)<1$ and $\|f(t, x, y)\| \leq c+d\|y\|$ if $\|x\| \leq R$.

In the third section of this paper, we establish an existence theorem for the system (1.1). To this aim, we introduce a notion of solution-tube of (1.1) which generalizes to systems the notions of lower and upper solutions introduced in $[1,2]$. This notion generalizes also condition (1.5) used by Henderson et al. [6] and Amster et al. [7]. Our notion of solutiontube is in the spirit of the notion of solution-tube for systems of second-order differential equations introduced in [10]. Our notion is new even in the case of systems of second-order difference equations. 
In the last section of this paper, we study the system (1.2). Again, we introduce a notion of solution-tube of (1.2) which generalizes the notion of lower and upper solutions used by Atici et al. [8]. This notion generalizes also condition (1.5) and the notion of solution-tube of systems of second-order differential equations introduced in [10]. In addition, we assume that $f$ satisfies a linear growth condition. It is worthwhile to mention that the time scale $\mathbb{T}$ does not need to be regular, and we do not require the restriction $d(\rho(b)-a)<1$ as in assumption (A2) used in [9].

Moreover, we point out that the right members of our systems are not necessarily continuous. Indeed, we assume that the weaker condition: $f$ is a $\Delta$-Carathéodory function. This condition is interesting in the case where the points of $\mathbb{T}$ are not all right scattered. We obtain the existence of solutions to (1.1) and to (1.2) in the Sobolev space $W_{\Delta}^{2,1}\left(\mathbb{T}, \mathbb{R}^{n}\right)$. To our knowledge, it is the first paper applying the theory of Sobolev spaces with topological methods to obtain solutions to (1.1) and (1.2). Solutions of second-order Hamiltonian systems on time scales were obtained in a Sobolev space via variational methods in [11]. Finally, let us mention that our results are new also in the continuous case and for systems of second-order difference equations.

\section{Preliminaries and Notations}

For sake of completeness, we recall some notations, definitions, and results concerning functions defined on time scales. The interested reader may consult $[12,13]$ and the references therein to find the proofs and to get a complete introduction to this subject.

Let $\mathbb{T}$ be a compact time scale with $a=\min \mathbb{T}<b=\max \mathbb{T}$. The forward jump operator $\sigma: \mathbb{T} \rightarrow \mathbb{T}$ (resp., the backward jump operator $\rho: \mathbb{T} \rightarrow \mathbb{T}$ ) is defined by

$$
\begin{gathered}
\sigma(t)= \begin{cases}\inf \{s \in \mathbb{T}: s>t\} & \text { if } t<b, \\
b & \text { if } t=b,\end{cases} \\
\left(\text { resp., } \rho(t)=\left\{\begin{array}{ll}
\sup \{s \in \mathbb{T}: s<t\} & \text { if } t>a, \\
a & \text { if } t=a
\end{array}\right) .\right.
\end{gathered}
$$

We say that $t<b$ is right scattered (resp., $t>a$ is left scattered) if $\sigma(t)>t$ (resp., $\rho(t)<t$ ) otherwise, we say that $t$ is right dense (resp., left dense). The set of right-scattered points of $\mathbb{T}$ is at most countable, see [14]. We denote it by

$$
R_{\mathbb{T}}:=\{t \in \mathbb{T}: t<\sigma(t)\}=\left\{t_{i}: i \in I\right\},
$$

for some $I \subset \mathbb{N}$. The graininess function $\mu: \mathbb{T} \rightarrow[0, \infty)$ is defined by $\mu(t)=\sigma(t)-t$. We denote

$$
\mathbb{T}^{\kappa}=\mathbb{T} \backslash(\rho(b), b], \quad \mathbb{T}_{0}=\mathbb{T} \backslash\{b\}
$$

So, $\mathbb{T}^{\mathcal{K}}=\mathbb{T}$ if $b$ is left dense, otherwise $\mathbb{T}^{\mathcal{K}}=\mathbb{T}_{0}$. Since $\mathbb{T}^{\mathcal{K}}$ is also a time scale, we denote

$$
\mathbb{T}^{\kappa^{2}}=\left(\mathbb{T}^{\kappa}\right)^{\kappa}, \quad \mathbb{T}_{0}^{\kappa^{2}}=\mathbb{T}^{\kappa^{2}} \backslash\{b\}
$$


In 1990, Hilger [15] introduced the concept of dynamic equations on time scales. This concept provides a unified approach to continuous and discrete calculus with the introduction of the notion of delta-derivative $x^{\Delta}(t)$. This notion coincides with $x^{\prime}(t)$ (resp., $\Delta x(t))$ in the case where the time scale $\mathbb{T}$ is an interval (resp., the discrete set $\{0,1, \ldots, N\}$ ).

Definition 2.1. A map $f: \mathbb{T} \rightarrow \mathbb{R}^{n}$ is $\Delta$-differentiable at $t \in \mathbb{T}^{\mathcal{K}}$ if there exists $f^{\Delta}(t) \in \mathbb{R}^{n}$ (called the $\Delta$-derivative of $f$ at $t$ ) such that for all $\epsilon>0$, there exists a neighborhood $U$ of $t$ such that

$$
\left\|\left(f(\sigma(t))-f(s)-f^{\Delta}(t)(\sigma(t)-s)\right)\right\| \leq \epsilon|\sigma(t)-s| \quad \forall s \in U
$$

We say that $f$ is $\Delta$-differentiable if $f^{\Delta}(t)$ exists for every $t \in \mathbb{T}^{\kappa}$.

If $f$ is $\Delta$-differentiable and if $f^{\Delta}$ is $\Delta$-differentiable at $t \in \mathbb{T}^{\kappa^{2}}$, we call $f^{\Delta \Delta}(t)=\left(f^{\Delta}\right)^{\Delta}(t)$ the second $\Delta$-derivative of $f$ at $t$.

Proposition 2.2. Let $f: \mathbb{T} \rightarrow \mathbb{R}^{n}$ and $t \in \mathbb{T}^{\kappa}$.

(i) If $f$ is $\Delta$-differentiable at $t$, then $f$ is continuous at $t$.

(ii) If $f$ is continuous at $t \in R_{\mathbb{T}}$, then

$$
f^{\Delta}(t)=\frac{f(\sigma(t))-f(t)}{\mu(t)} .
$$

(iii) The map $f$ is $\Delta$-differentiable at $t \in \mathbb{T}^{\mathcal{K}} \backslash R_{\mathbb{T}}$ if and only if

$$
f^{\Delta}(t)=\lim _{s \rightarrow t} \frac{f(t)-f(s)}{t-s}
$$

Proposition 2.3. If $f: \mathbb{T} \rightarrow \mathbb{R}^{n}$ and $g: \mathbb{T} \rightarrow \mathbb{R}^{m}$ are $\Delta$-differentiable at $t \in \mathbb{T}^{\kappa}$, then

(i) if $n=m,(\alpha f+g)^{\Delta}(t)=\alpha f^{\Delta}(t)+g^{\Delta}(t)$ for every $\alpha \in \mathbb{R}$,

(ii) if $m=1,(f g)^{\Delta}(t)=g(t) f^{\Delta}(t)+f(\sigma(t)) g^{\Delta}(t)=f(t) g^{\Delta}(t)+g(\sigma(t)) f^{\Delta}(t)$,

(iii) if $m=1$ and $g(t) g(\sigma(t)) \neq 0$, then

$$
\left(\frac{f}{g}\right)^{\Delta}(t)=\frac{g(t) f^{\Delta}(t)-f(t) g^{\Delta}(t)}{g(t) g(\sigma(t))}
$$

(iv) if $W \subset \mathbb{R}^{n}$ is open and $h: W \rightarrow \mathbb{R}$ is differentiable at $f(t) \in W$ and $t \notin R_{\mathbb{T}}$, then $(h \circ f)^{\Delta}(t)=\left\langle h^{\prime}(f(t)), f^{\Delta}(t)\right\rangle$.

We denote $C\left(\mathbb{T}, \mathbb{R}^{n}\right)$ the space of continuous maps on $\mathbb{T}$, and we denote $C^{1}\left(\mathbb{T}, \mathbb{R}^{n}\right)$ the space of continuous maps on $\mathbb{T}$ with continuous $\Delta$-derivative on $\mathbb{T}^{\kappa}$. With the norm $\|x\|_{0}=$ $\max \{\|x(t)\|: t \in \mathbb{T}\}$ (resp., $\left.\|x\|_{1}=\max \left\{\|x\|_{0}, \max \left\{\left\|x^{\Delta}(t)\right\|: t \in \mathbb{T}^{\kappa}\right\}\right\}\right), C\left(\mathbb{T}, \mathbb{R}^{n}\right)$ (resp., $\left.C^{1}\left(\mathbb{T}, \mathbb{R}^{n}\right)\right)$ is a Banach space. 
We study the second $\Delta$-derivative of the norm of a map.

Lemma 2.4. Let $x: \mathbb{T} \rightarrow \mathbb{R}^{n}$ be $\Delta$-differentiable.

(1) On $\left\{t \in \mathbb{T}^{\kappa^{2}}:\|x(\sigma(t))\|>0\right.$ and $x^{\Delta \Delta}(t)$ exists $\}$,

$$
\|x(t)\|^{\Delta \Delta} \geq \frac{\left\langle x(\sigma(t)), x^{\Delta \Delta}(t)\right\rangle}{\|x(\sigma(t))\|} .
$$

(2) On $\left\{t \in \mathbb{T}^{\kappa^{2}} \backslash R_{\mathbb{T}}:\|x(\sigma(t))\|>0\right.$ and $x^{\Delta \Delta}(t)$ exists $\}$,

$$
\|x(t)\|^{\Delta \Delta}=\frac{\left\langle x(t), x^{\Delta \Delta}(t)\right\rangle+\left\|x^{\Delta}(t)\right\|^{2}}{\|x(t)\|}-\frac{\left\langle x(t), x^{\Delta}(t)\right\rangle^{2}}{\|x(t)\|^{3}} .
$$

Proof. Denote $A=\left\{t \in \mathbb{T}^{\kappa^{2}}:\|x(\sigma(t))\|>0\right.$ and $x^{\Delta \Delta}(t)$ exists $\}$. By Proposition 2.3, on the set $A \backslash R_{\mathbb{T}}$, we have

$$
\begin{gathered}
\|x(t)\|^{\Delta}=\frac{\left\langle x(t), x^{\Delta}(t)\right\rangle}{\|x(t)\|}, \\
\|x(t)\|^{\Delta \Delta}=\frac{\left\langle x(t), x^{\Delta \Delta}(t)\right\rangle+\left\|x^{\Delta}(t)\right\|^{2}}{\|x(t)\|}-\frac{\left\langle x(t), x^{\Delta}(t)\right\rangle^{2}}{\|x(t)\|^{3}} \geq \frac{\left\langle x(\sigma(t)), x^{\Delta \Delta}(t)\right\rangle}{\|x(\sigma(t))\|} .
\end{gathered}
$$

If $t \in A$ is such that $t<\sigma(t)=\sigma^{2}(t)$, then by Propositions 2.2 and 2.3, we have

$$
\begin{aligned}
\|x(t)\|^{\Delta \Delta} & =\frac{\|x(\sigma(t))\|^{\Delta}-\|x(t)\|^{\Delta}}{\mu(t)} \\
& =\frac{\left\langle x(\sigma(t)), x^{\Delta}(\sigma(t))\right\rangle}{\mu(t)\|x(\sigma(t))\|}-\frac{\|x(\sigma(t))\|-\|x(t)\|}{\mu(t)^{2}} \\
& =\frac{\left\langle x(\sigma(t)), x^{\Delta}(t)+\mu(t) x^{\Delta \Delta}(t)\right\rangle}{\mu(t)\|x(\sigma(t))\|}-\frac{\left\langle x(\sigma(t)), x(t)+\mu(t) x^{\Delta}(t)\right\rangle}{\mu(t)^{2}\|x(\sigma(t))\|}+\frac{\|x(t)\|}{\mu(t)^{2}} \\
& =\frac{\left\langle x(\sigma(t)), x^{\Delta \Delta}(t)\right\rangle}{\|x(\sigma(t))\|}-\frac{\langle x(\sigma(t)), x(t)\rangle}{\mu(t)^{2}\|x(\sigma(t))\|}+\frac{\|x(t)\|}{\mu(t)^{2}} \\
& \geq \frac{\left\langle x(\sigma(t)), x^{\Delta \Delta}(t)\right\rangle}{\|x(\sigma(t))\|} .
\end{aligned}
$$


If $t \in A$ is such that $t<\sigma(t)<\sigma^{2}(t)$, then

$$
\begin{aligned}
\|x(t)\|^{\Delta \Delta} & =\frac{\|x(\sigma(t))\|^{\Delta}-\|x(t)\|^{\Delta}}{\mu(t)} \\
& =\frac{\left\|x\left(\sigma^{2}(t)\right)\right\|-\|x(\sigma(t))\|}{\mu(\sigma(t)) \mu(t)}-\frac{\|x(\sigma(t))\|-\|x(t)\|}{\mu(t)^{2}} \\
& \geq \frac{\left\langle x(\sigma(t)), x\left(\sigma^{2}(t)\right)-x(\sigma(t))\right\rangle}{\mu(\sigma(t)) \mu(t)\|x(\sigma(t))\|}-\frac{\|x(\sigma(t))\|-\|x(t)\|}{\mu(t)^{2}} \\
& =\frac{\left\langle x(\sigma(t)), x^{\Delta}(\sigma(t))\right\rangle}{\mu(t)\|x(\sigma(t))\|}-\frac{\|x(\sigma(t))\|-\|x(t)\|}{\mu(t)^{2}},
\end{aligned}
$$

and we conclude as in the previous case.

Let $\varepsilon>0$. The exponential function $e_{\varepsilon}\left(\cdot, t_{0}\right)$ is defined by

$$
e_{\varepsilon}\left(t, t_{0}\right)=\exp \left(\int_{\left[t_{0}, t\right) \cap \mathbb{T}} \xi_{\varepsilon}(\mu(s)) \Delta s\right)
$$

where

$$
\xi_{\varepsilon}(h)= \begin{cases}\varepsilon, & \text { if } h=0 \\ \frac{\log (1+h \varepsilon)}{h}, & \text { if } h>0 .\end{cases}
$$

It is the unique solution to the initial value problem

$$
x^{\Delta}(t)=\varepsilon x(t), \quad x\left(t_{0}\right)=1 .
$$

Here is a result on time scales, analogous to Gronwall's inequality. The reader may find the proof of this result in [13].

Theorem 2.5. Let $\alpha>0, \varepsilon>0$, and $y \in C(\mathbb{T}, \mathbb{R})$. If

$$
y(t) \leq \alpha+\int_{[a, t) \cap \mathbb{T}} \varepsilon y(s) \Delta s \quad \text { for every } t \in \mathbb{T}
$$

then

$$
y(t) \leq \alpha e_{\varepsilon}(t, a) \quad \text { for every } t \in \mathbb{T} \text {. }
$$

We recall some notions and results related to the theory of $\Delta$-measure and $\Delta$-Lebesgue integration introduced by Bohner and Guseinov in [12]. The reader is also referred to [14] 
for expressions of the $\Delta$-measure and the $\Delta$-integral in terms of the classical Lebesgue measure and the classical Lebesgue integral, respectively.

Definition 2.6. A set $A \subset \mathbb{T}$ is said to be $\Delta$-measurable if for every set $E \subset \mathbb{T}$,

$$
m_{1}^{*}(E)=m_{1}^{*}(E \cap A)+m_{1}^{*}(E \cap(\mathbb{T} \backslash A)),
$$

where

$$
m_{1}^{*}(E)= \begin{cases}\inf \left\{\sum_{k=1}^{m}\left(d_{k}-c_{k}\right): E \subset \bigcup_{k=1}^{m}\left[c_{k}, d_{k}\right) \text { with } c_{k}, d_{k} \in \mathbb{T}\right\} & \text { if } b \notin E \\ \infty & \text { if } b \in E\end{cases}
$$

The $\Delta$-measure on $\mathcal{M}\left(m_{1}^{*}\right):=\{A \subset \mathbb{T}: A$ is $\Delta$-measurable $\}$, denoted by $\mu_{\Delta}$, is the restriction of $m_{1}^{*}$ to $\mathcal{M}\left(m_{1}^{*}\right)$. So, $\left(\mathbb{T}, \mathcal{M}\left(m_{1}^{*}\right), \mu_{\Delta}\right)$ is a complete measurable space.

Proposition 2.7 (see [14]). Let $A \subset \mathbb{T}$, then $A$ is $\Delta$-measurable if and only if $A$ is Lebesgue measurable. Moreover, if $b \notin A$,

$$
\mu_{\Delta}(A)=m(A)+\sum_{t_{i} \in A \cap R_{\mathbb{T}}}\left(\sigma\left(t_{i}\right)-t_{i}\right)
$$

where $m$ is the Lebesgue measure.

The notions of $\Delta$-measurable and $\Delta$-integrable functions $f: \mathbb{T} \rightarrow \mathbb{R}^{n}$ can be defined similarly to the general theory of Lebesgue integral.

Let $E \subset \mathbb{T}$ be a $\Delta$-measurable set and $f: \mathbb{T} \rightarrow \mathbb{R}^{n}$ a $\Delta$-measurable function. We say that $f \in L_{\Delta}^{1}\left(E, \mathbb{R}^{n}\right)$ provided

$$
\int_{E}\|f(s)\| \Delta s<\infty
$$

The set $L_{\Delta}^{1}\left(\mathbb{T}_{0}, \mathbb{R}^{n}\right)$ is a Banach space endowed with the norm

$$
\|f\|_{L_{\Delta}^{1}}:=\int_{\mathbb{T}_{0}}\|f(s)\| \Delta s
$$

Here is an analog of the Lebesgue dominated convergence Theorem which can be proved as in the general theory of Lebesgue integration theory.

Theorem 2.8. Let $\left\{f_{k}\right\}_{k \in \mathbb{N}}$ be a sequence of functions in $L_{\Delta}^{1}\left(\mathbb{T}_{0}, \mathbb{R}^{n}\right)$. Assume that there exists a function $f: \mathbb{T}_{0} \rightarrow \mathbb{R}^{n}$ such that $f_{k}(t) \rightarrow f(t) \Delta$-a.e. $t \in \mathbb{T}_{0}$, and there exists a function $g \in L_{\Delta}^{1}\left(\mathbb{T}_{0}\right)$ such that $\left\|f_{k}(t)\right\| \leq g(t) \Delta$-a.e. $t \in \mathbb{T}_{0}$ and for every $k \in \mathbb{N}$, then $f_{k} \rightarrow f$ in $L_{\Delta}^{1}\left(\mathbb{T}_{0}, \mathbb{R}^{n}\right)$.

In our existence results, we will consider $\Delta$-Carathéodory functions. 
Definition 2.9. A function $f: \mathbb{T}_{0} \times \mathbb{R}^{m} \rightarrow \mathbb{R}^{n}$ is $\Delta$-Carathéodory if the following conditions hold:

(i) $t \mapsto f(t, x)$ is $\Delta$-measurable for every $x \in \mathbb{R}^{m}$,

(ii) $x \mapsto f(t, x)$ is continuous for $\Delta$-almost every $t \in \mathbb{T}_{0}$,

(iii) for every $r>0$, there exists $h_{r} \in L_{\Delta}^{1}\left(\mathbb{T}_{0},[0, \infty)\right)$ such that $\|f(t, x)\| \leq h_{r}(t)$ for $\Delta$-almost every $t \in \mathbb{T}_{0}$ and for every $x \in \mathbb{R}^{m}$ such that $\|x\| \leq r$.

In this context, there is also a notion of absolute continuity introduced in [16].

Definition 2.10. A function $f: \mathbb{T} \rightarrow \mathbb{R}^{n}$ is said to be absolutely continuous on $\mathbb{T}$ if for every $\epsilon>0$, there exists a $\delta>0$ such that if $\left\{\left[a_{k}, b_{k}\right)\right\}_{k=1}^{m}$ with $a_{k}, b_{k} \in \mathbb{T}$ is a finite pairwise disjoint family of subintervals satisfying

$$
\sum_{k=1}^{m}\left(b_{k}-a_{k}\right)<\delta, \quad \text { then } \sum_{k=1}^{m}\left\|f\left(b_{k}\right)-f\left(a_{k}\right)\right\|<\epsilon .
$$

Proposition 2.11 (see [17]). If $f: \mathbb{T} \rightarrow \mathbb{R}^{n}$ is an absolutely continuous function, then the $\Delta$-measure of the set $\left\{t \in \mathbb{T}_{0} \backslash R_{\mathbb{T}}: f(t)=0\right.$ and $\left.f^{\Delta}(t) \neq 0\right\}$ is zero.

Proposition 2.12 (see [17]). If $g \in L_{\Delta}^{1}\left(\mathbb{T}_{0}, \mathbb{R}^{n}\right)$ and $f: \mathbb{T} \rightarrow \mathbb{R}^{n}$ is the function defined by

$$
f(t):=\int_{[a, t) \cap \mathbb{T}} g(s) \Delta s
$$

then $f$ is absolutely continuous and $f^{\Delta}(t)=g(t) \Delta$-almost everywhere on $\mathbb{T}_{0}$.

Proposition 2.13 (see [16]). A function $f: \mathbb{T} \rightarrow \mathbb{R}$ is absolutely continuous on $\mathbb{T}$ if and only if $f$ is $\Delta$-differentiable $\Delta$-almost everywhere on $\mathbb{T}_{0}, f^{\Delta} \in L_{\Delta}^{1}\left(\mathbb{T}_{0}\right)$ and

$$
\int_{[a, t) \cap \mathbb{T}} f^{\Delta}(s) \Delta s=f(t)-f(a), \quad \text { for every } t \in \mathbb{T} .
$$

We also recall a notion of Sobolev space, see [18],

$$
\begin{gathered}
W_{\Delta}^{1,1}\left(\mathbb{T}, \mathbb{R}^{n}\right)=\left\{x \in L_{\Delta}^{1}\left(\mathbb{T}_{0}, \mathbb{R}^{n}\right): \exists g \in L_{\Delta}^{1}\left(\mathbb{T}_{0}, \mathbb{R}^{n}\right) \text { such that } \int_{\mathbb{T}_{0}} x(s) \phi^{\Delta}(s) \Delta s\right. \\
\left.=-\int_{\mathbb{T}_{0}} g(s) \phi(\sigma(s)) \Delta s \text { for every } \phi \in C_{0, \mathrm{rd}}^{1}(\mathbb{T})\right\}
\end{gathered}
$$


where

$$
\begin{aligned}
C_{0, \mathrm{rd}}^{1}(\mathbb{T})=\{\phi: \mathbb{T} \rightarrow \mathbb{R}: \phi(a)=0=\phi(b), \phi \text { is } \Delta \text {-differentiable and } \\
\phi^{\Delta} \text { is continuous at right-dense points of } \mathbb{T} \text { and } \\
\text { its left-sided limits exist at left-dense points of } \mathbb{T}\} .
\end{aligned}
$$

A function $x \in W_{\Delta}^{1,1}\left(\mathbb{T}, \mathbb{R}^{n}\right)$ can be identified to an absolutely continuous map.

Proposition 2.14 (see [18]). Suppose that $x \in W_{\Delta}^{1,1}(\mathbb{T}, \mathbb{R})$ with some $g \in L_{\Delta}^{1}\left(\mathbb{T}_{0}, \mathbb{R}\right)$ satisfying (2.27), then there exists $y: \mathbb{T} \rightarrow \mathbb{R}$ absolutely continuous such that

$$
y=x, \quad y^{\Delta}=g \quad \Delta \text {-a.e. on } \mathbb{T}_{0} .
$$

Moreover, if $g$ is $C_{\mathrm{rd}}\left(\mathbb{T}^{\kappa}, \mathbb{R}\right)$, then there exists $y \in C_{\mathrm{rd}}^{1}(\mathbb{T}, \mathbb{R})$ such that

$$
y=x \quad \Delta \text {-a.e. on } \mathbb{T}_{0}, \quad y^{\Delta}=g \quad \Delta \text {-a.e. on } \mathbb{T}^{\kappa} .
$$

Sobolev spaces of higher order can be defined inductively as follows:

$$
W_{\Delta}^{2,1}\left(\mathbb{T}, \mathbb{R}^{n}\right)=\left\{x \in W_{\Delta}^{1,1}\left(\mathbb{T}, \mathbb{R}^{n}\right): x^{\Delta} \in W_{\Delta}^{1,1}\left(\mathbb{T}^{\kappa}, \mathbb{R}^{n}\right)\right\}
$$

With the norm $\|x\|_{W_{\Delta}^{1,1}}:=\|x\|_{L_{\Delta}^{1}}+\left\|x^{\Delta}\right\|_{L_{\Delta}^{1}}$ (resp., $\left.\|x\|_{W_{\Delta}^{2,1}}:=\|x\|_{L_{\Delta}^{1}}+\left\|x^{\Delta}\right\|_{L_{\Delta}^{1}}+\left\|x^{\Delta \Delta}\right\|_{L_{\Delta}^{1}}\right)$, $W_{\Delta}^{1,1}\left(\mathbb{T}, \mathbb{R}^{n}\right)\left(\right.$ resp., $\left.W_{\Delta}^{2,1}\left(\mathbb{T}, \mathbb{R}^{n}\right)\right)$ is a Banach space.

Remark 2.15. By Proposition 2.7, we know that $\mu_{\Delta}(\{t\})>0$ for every $t \in R_{\mathbb{T}}$. From this fact and the previous proposition, one realizes that there is no interest to look for solutions to (1.1) and (1.2) in $W_{\Delta}^{2,1}\left(\mathbb{T}, \mathbb{R}^{n}\right)$ and to consider $\Delta$-Carathéodory maps $f$ in the case where the time scale is such that $\mu_{\Delta}\left(\mathbb{T}_{0} \backslash R_{\mathbb{T}}\right)=0$. In particular, this is the case for difference equations. Let us point out that we consider more general time scales. Nevertheless, the results that we obtained are new in both cases.

As in the classical case, some embeddings have nice properties.

Proposition 2.16 (see [18]). The inclusion $j_{1}: W_{\Delta}^{2,1}\left(\mathbb{T}, \mathbb{R}^{n}\right) \rightarrow C^{1}\left(\mathbb{T}, \mathbb{R}^{n}\right)$ is continuous.

Proposition 2.17. The inclusion $j_{0}: W_{\Delta}^{2,1}\left(\mathbb{T}, \mathbb{R}^{n}\right) \hookrightarrow C\left(\mathbb{T}, \mathbb{R}^{n}\right)$ is a continuous, compact, linear operator.

Proof. Arguing as in the proof of the Arzelà-Ascoli Theorem, we can show that the inclusion $i: C^{1}\left(\mathbb{T}, \mathbb{R}^{n}\right) \hookrightarrow C\left(\mathbb{T}, \mathbb{R}^{n}\right)$ is linear, continuous, and compact. The conclusion follows from the previous proposition since $j_{0}=i \circ j_{1}$.

We obtain a maximum principle in this context. To this aim, we use the following result. 
Lemma 2.18 (see [19]). Let $f: \mathbb{T} \rightarrow \mathbb{R}$ be a function with a local maximum at $t_{0} \in(a, b) \cap \mathbb{T}$. If $f^{\Delta \Delta}\left(\rho\left(t_{0}\right)\right)$ exists, then $f^{\Delta \Delta}\left(\rho\left(t_{0}\right)\right) \leq 0$ provided $t_{0}$ is not at the same time left dense and right scattered.

Theorem 2.19. Let $r \in W_{\Delta}^{2,1}(\mathbb{T})$ be a function such that $r^{\Delta \Delta}(t)>0 \Delta$-almost everywhere on $\{t \in$ $\left.\mathbb{T}_{0}^{\kappa^{2}}: r(\sigma(t))>0\right\}$. If one of the following conditions holds:

(i) $a_{0} r(a)-\gamma_{0} r^{\Delta}(a) \leq 0$ and $a_{1} r(b)+\gamma_{1} r^{\Delta}(\rho(b)) \leq 0$ (where $a_{0}, a_{1}, \gamma_{0}$, and $\gamma_{1}$ are defined as in (1.4)),

(ii) $r(a)=r(b)$ and $r^{\Delta}(a) \geq r^{\Delta}(\rho(b))$,

then $r(t) \leq 0$, for every $t \in \mathbb{T}$.

Proof. If the conclusion is false, there exists $t_{0} \in \mathbb{T}$ such that

$$
r\left(t_{0}\right)=\max _{t \in \mathbb{T}} r(t)>0
$$

In the case where $a \leq \rho\left(t_{0}\right)<t_{0}<b$, then $r^{\Delta \Delta}\left(\rho\left(t_{0}\right)\right)$ exists since $\mu_{\Delta}\left(\left\{\rho\left(t_{0}\right)\right\}\right)=t_{0}-$ $\rho\left(t_{0}\right)>0$ and $r \in W_{\Delta}^{2,1}(\mathbb{T})$. By Lemma 2.18, $r^{\Delta \Delta}\left(\rho\left(t_{0}\right)\right) \leq 0$, which is a contradiction since $r\left(\sigma\left(\rho\left(t_{0}\right)\right)\right)=r\left(t_{0}\right)>0$.

If $a<\rho\left(t_{0}\right)=t_{0}=\sigma\left(t_{0}\right)<b$, there exists $t_{1}>t_{0}$ such that $r(\sigma(t))>0$ for every $t \in\left(t_{0}, t_{1}\right) \cap \mathbb{T}$. On the other hand, since $r\left(t_{0}\right)$ is a maximum, $r^{\Delta}\left(t_{0}\right)=0$, and there exists $s \in\left(t_{0}, t_{1}\right)$ such that $r^{\Delta}(s) \leq 0$, thus,

$$
0 \geq r^{\Delta}(s)-r^{\Delta}\left(t_{0}\right)=\int_{\left[t_{0}, s\right) \cap \mathbb{T}} r^{\Delta \Delta}(\tau) \Delta \tau>0,
$$

by hypothesis and Proposition 2.13, which is a contradiction. Observe that the same argument applies if $a=\rho\left(t_{0}\right)=t_{0}=\sigma\left(t_{0}\right)$ and $r^{\Delta}\left(t_{0}\right)=0$. Notice also that if $\rho\left(t_{0}\right)=t_{0}=\sigma\left(t_{0}\right)=b$ and $r^{\Delta}\left(t_{0}\right)=0$, we get a contradiction with an analogous argument for a suitable $t_{1}<t_{0}$.

If $a \leq \rho\left(t_{0}\right)=t_{0}<\sigma\left(t_{0}\right)<b$ and $r\left(t_{0}\right)=r\left(\sigma\left(t_{0}\right)\right)$, we argue as in the first case replacing $t_{0}$ by $\sigma\left(t_{0}\right)$ to obtain a contradiction.

In the case where $a<\rho\left(t_{0}\right)=t_{0}<\sigma\left(t_{0}\right) \leq b$ and $r\left(t_{0}\right)>r\left(\sigma\left(t_{0}\right)\right)$, then $r^{\Delta}\left(t_{0}\right)<0$. Since $t \mapsto r^{\Delta}(t)$ is continuous, there exists $\delta>0$ such that $r^{\Delta}(t)<0$ on an interval $\left(t_{0}-\delta, t_{0}\right)$, which contradicts the maximality of $r\left(t_{0}\right)$.

Observe that $a=t_{0}$ and $r^{\Delta}(a)<0$ could happen if $a=t_{0}=\sigma\left(t_{0}\right)$ or if $a=t_{0}<\sigma\left(t_{0}\right)$ and $r(a)>r(\sigma(a))$. In this case, we get a contradiction if $r$ satisfies condition (i). On the other hand, if $r$ satisfies condition (ii), then $r(b)=r(a)>0$ and $r^{\Delta}(\rho(b))<0$. So $\rho(b)=b$ since $r(b) \geq r(\rho(b))$. This contradicts the maximality of $r(b)$.

On the other hand, the case where $t_{0}=b$ and $r$ satisfies condition (i) can be treated similarly to the previous case. consider

Finally, we define the $\Delta$-differential operator associated to the problems that we will

$$
L: W_{\Delta, B}^{2,1}\left(\mathbb{T}, \mathbb{R}^{n}\right) \longrightarrow L_{\Delta}^{1}\left(\mathbb{T}_{0}^{\kappa}, \mathbb{R}^{n}\right) \text { defined by } L(x)(t):=x^{\Delta \Delta}(t)-x(\sigma(t)),
$$


where $W_{\Delta, B}^{2,1}\left(\mathbb{T}, \mathbb{R}^{n}\right):=\left\{x \in W_{\Delta}^{2,1}\left(\mathbb{T}, \mathbb{R}^{n}\right): x \in(\mathrm{BC})\right\}$ with $(\mathrm{BC})$ denoting the periodic boundary condition (1.3) or the Sturm-Liouville boundary condition (1.4).

Proposition 2.20. The operator $L$ is invertible and $L^{-1}$ is affine and continuous.

Proof. If (BC) denotes the Sturm-Liouville boundary condition (1.4), consider the associated homogeneous boundary condition

$$
\begin{gathered}
a_{0} x(a)-\gamma_{0} x^{\Delta}(a)=0, \\
a_{1} x(b)+\gamma_{1} x^{\Delta}(\rho(b))=0 .
\end{gathered}
$$

Denote

$$
W_{\Delta, B_{0}}^{2,1}\left(\mathbb{T}, \mathbb{R}^{n}\right)= \begin{cases}\left\{x \in W_{\Delta}^{2,1}\left(\mathbb{T}, \mathbb{R}^{n}\right): x \text { satisfies (1.3) }\right\} & \text { if (BC) denotes (1.3) } \\ \left\{x \in W_{\Delta}^{2,1}\left(\mathbb{T}, \mathbb{R}^{n}\right): x \text { satisfies (2.35) }\right\} & \text { if (BC) denotes (1.4). }\end{cases}
$$

Notice that $W_{\Delta, B_{0}}^{2,1}\left(\mathbb{T}, \mathbb{R}^{n}\right)$ is a Banach space. Define

$$
L_{0}: W_{\Delta, B_{0}}^{2,1}\left(\mathbb{T}, \mathbb{R}^{n}\right) \longrightarrow L_{\Delta}^{1}\left(\mathbb{T}_{0}^{\kappa}, \mathbb{R}^{n}\right) \text { by } L_{0}(x)(t):=x^{\Delta \Delta}(t)-x(\sigma(t))
$$

It is obvious that $L_{0}$ is linear and continuous. It follows directly from Theorem 2.19 that $L_{0}$ is injective.

If (BC) denotes (1.4) (resp., (1.3)), let $G(t, s)$ be the Green function given in [13, Theorem 4.70] (resp., [13, Theorem 4.89]). Arguing as in [13, Theorem 4.70] (resp., [13, Theorem 4.89]), one can verify that for any $h \in L_{\Delta}^{1}\left(\mathbb{T}_{0}^{\kappa}, \mathbb{R}^{n}\right)$,

$$
x(t)=\int_{[a, \rho(b)) \cap \mathbb{T}} G(t, s) h(s) \Delta s
$$

is a solution of $L_{0}(x)=h$. So, $L_{0}$ is bijective and, hence, invertible with $L_{0}^{-1}$ continuous by the inverse mapping theorem.

Finally, if (BC) denotes (1.3), since $L=L_{0}$, we have the conclusion. On the other hand, if (BC) denotes (1.4), let $y$ be given in [13, Theorem 4.67] such that

$$
y^{\Delta \Delta}(t)-y(\sigma(t))=0 \quad \text { on } \mathbb{T}^{\kappa^{2}}, \quad a_{0} y(a)-\gamma_{0} y^{\Delta}(a)=x_{0}, \quad a_{1} y(b)+\gamma_{1} y^{\Delta}(\rho(b))=x_{1}
$$

then $L^{-1}=y+L_{0}^{-1}$.

Remark 2.21. We could have considered the operator

$$
\tilde{L}: W_{\Delta, B}^{2,1}\left(\mathbb{T}, \mathbb{R}^{n}\right) \longrightarrow L_{\Delta}^{1}\left(\mathbb{T}_{0}^{\kappa}, \mathbb{R}^{n}\right) \text { defined by } \tilde{L}(x)(t):=x^{\Delta \Delta}(t),
$$


when the boundary condition is (1.4) with suitable constants $a_{0}, a_{1}, \gamma_{0}, \gamma_{1}$ such that $\tilde{L}$ is injective. For simplicity, we prefer to use only the operator $L$.

\section{Nonlinearity Not Depending on the Delta-Derivative}

In this section, we establish existence results for the problem

$$
x^{\Delta \Delta}(t)=f(t, x(\sigma(t))), \quad \Delta \text {-a.e. } t \in \mathbb{T}_{0}^{\kappa^{2}}, x \in(\mathrm{BC}),
$$

where $(\mathrm{BC})$ denotes the periodic boundary condition

$$
\begin{gathered}
x(a)=x(b), \\
x^{\Delta}(a)=x^{\Delta}(\rho(b)),
\end{gathered}
$$

or the Sturm-Liouville boundary condition

$$
\begin{array}{r}
a_{0} x(a)-\gamma_{0} x^{\Delta}(a)=x_{0}, \\
a_{1} x(b)+\gamma_{1} x^{\Delta}(\rho(b))=x_{1},
\end{array}
$$

where $a_{0}, a_{1}, \gamma_{0}, \gamma_{1} \geq 0, \max \left\{a_{0}, \gamma_{0}\right\}>0$, and $\max \left\{a_{1}, \gamma_{1}\right\}>0$. We look for solutions in $W_{\Delta}^{2,1}\left(\mathbb{T}, \mathbb{R}^{n}\right)$.

We introduce the notion of solution-tube for the problem (3.1).

Definition 3.1. Let $(v, M) \in W_{\Delta}^{2,1}\left(\mathbb{T}, \mathbb{R}^{n}\right) \times W_{\Delta}^{2,1}(\mathbb{T},[0, \infty))$. We say that $(v, M)$ is a solution-tube of (3.1) if

(i) $\left\langle x-v(\sigma(t)), f(t, x)-v^{\Delta \Delta}(t)\right\rangle \geq M(\sigma(t)) M^{\Delta \Delta}(t)$ for $\Delta$-almost every $t \in \mathbb{T}_{0}^{\kappa^{2}}$ and for every $x \in \mathbb{R}^{n}$ such that $\|x-v(\sigma(t))\|=M(\sigma(t))$,

(ii) $v^{\Delta \Delta}(t)=f(t, v(\sigma(t)))$ and $M^{\Delta \Delta}(t) \leq 0$ for $\Delta$-almost every $t \in \mathbb{T}_{0}^{\kappa^{2}}$ such that $M(\sigma(t))=0$,

(iii) (a) if (BC) denotes (3.2), then $v(a)=v(b), M(a)=M(b)$, and $\left\|v^{\Delta}(\rho(b))-v^{\Delta}(a)\right\| \leq$ $M^{\Delta}(\rho(b))-M^{\Delta}(a)$,

(b) if (BC) denotes (3.3), $\left\|x_{0}-\left(a_{0} v(a)-\gamma_{0} v^{\Delta}(a)\right)\right\| \leq a_{0} M(a)-\gamma_{0} M^{\Delta}(a)$, $\left\|x_{1}-\left(a_{1} v(b)+\gamma_{1} v^{\Delta}(\rho(b))\right)\right\| \leq a_{1} M(b)+\gamma_{1} M^{\Delta}(\rho(b))$.

We denote

$$
T(v, M)=\left\{x \in W_{\Delta}^{2,1}\left(\mathbb{T}, \mathbb{R}^{n}\right):\|x(t)-v(t)\| \leq M(t) \forall t \in \mathbb{T}\right\}
$$

We state the main theorem of this section. 
Theorem 3.2. Let $f: \mathbb{T}_{0}^{\kappa} \times \mathbb{R}^{n} \rightarrow \mathbb{R}^{n}$ be a $\Delta$-Carathéodory function. If $(v, M) \in W_{\Delta}^{2,1}\left(\mathbb{T}, \mathbb{R}^{n}\right) \times$ $W_{\Delta}^{2,1}(\mathbb{T},[0, \infty))$ is a solution-tube of (3.1), then the system (3.1) has a solution $x \in W_{\Delta}^{2,1}\left(\mathbb{T}, \mathbb{R}^{n}\right) \cap$ $T(v, M)$.

In order to prove this result, we consider the following modified problem:

$$
\begin{gathered}
x^{\Delta \Delta}(t)-x(\sigma(t))=f(t, \bar{x}(\sigma(t)))-\bar{x}(\sigma(t)), \quad \Delta \text {-a.e. } t \in \mathbb{T}_{0}^{\kappa^{2}}, \\
x \in(\mathrm{BC}),
\end{gathered}
$$

where

$$
\bar{x}(s)= \begin{cases}\frac{M(s)}{\|x-v(s)\|}(x-v(s))+v(s) & \text { if }\|x-v(s)\|>M(s) \\ x & \text { otherwise. }\end{cases}
$$

We define the operator $F: C\left(\mathbb{T}, \mathbb{R}^{n}\right) \rightarrow L_{\Delta}^{1}\left(\mathbb{T}_{0}^{\kappa}, \mathbb{R}^{n}\right)$ by

$$
F(x)(t):=f(t, \bar{x}(\sigma(t)))-\bar{x}(\sigma(t))
$$

Proposition 3.3. Under the assumptions of Theorem 3.2, the operator F defined above is continuous and bounded.

Proof. First of all, we show that the set $F\left(C\left(\mathbb{T}, \mathbb{R}^{n}\right)\right)$ is bounded. Fix $R>\|v\|_{0}+\|M\|_{0}$. Let $h_{R} \in L_{\Delta}^{1}\left(\mathbb{T}_{0}^{\kappa},[0, \infty)\right)$ be given by Definition 2.9(iii). Thus, for every $x \in C\left(\mathbb{T}, \mathbb{R}^{n}\right)$,

$$
\|F(x)(s)\| \leq h_{R}(s)+R=: h(s) \quad \Delta \text {-a.e. } s \in \mathbb{T}_{0}^{\kappa} .
$$

To prove the continuity of $F$, we consider $\left\{x_{k}\right\}_{k \in \mathbb{N}}$ a sequence of $C\left(\mathbb{T}, \mathbb{R}^{n}\right)$ converging to $x \in C\left(\mathbb{T}, \mathbb{R}^{n}\right)$. We already know that for every $k \in \mathbb{N}$,

$$
\left\|F\left(x_{k}\right)(s)\right\| \leq h(s) \quad \Delta \text {-a.e. } s \in \mathbb{T}_{0}^{\kappa} .
$$

One can easily check that $\bar{x}_{n}(t) \rightarrow \bar{x}(t)$ for all $t \in \mathbb{T}$. It follows from Definition 2.9(ii) that

$$
F\left(x_{k}\right)(s) \longrightarrow F(x)(s) \quad \Delta \text {-a.e. } s \in \mathbb{T}_{0}^{\kappa}
$$

Theorem 2.8 implies that $F\left(x_{k}\right) \rightarrow F(x)$ in $L_{\Delta}^{1}\left(\mathbb{T}_{0}^{\kappa}, \mathbb{R}^{n}\right)$.

Lemma 3.4. Under the assumptions of Theorem 3.2, every solution $x$ of $(3.5)$ is in $T(v, M)$.

Proof. Since $x \in W_{\Delta}^{2,1}\left(\mathbb{T}, \mathbb{R}^{n}\right)$ (resp., $v \in W_{\Delta}^{2,1}\left(\mathbb{T}, \mathbb{R}^{n}\right), M \in W_{\Delta}^{2,1}(\mathbb{T}, \mathbb{R})$ ), $x^{\Delta \Delta}(t)$ (resp., $v^{\Delta \Delta}(t)$, $\left.M^{\Delta \Delta}(t)\right)$, there exists $\Delta$-almost everywhere on $\mathbb{T}^{\kappa^{2}}$. Denote

$$
A=\left\{t \in \mathbb{T}_{0}^{\kappa^{2}}:\|x(\sigma(t))-v(\sigma(t))\|>M(\sigma(t))\right\} .
$$


By Lemma 2.4(1),

$$
(\|x(t)-v(t)\|-M(t))^{\Delta \Delta} \geq \frac{\left\langle x(\sigma(t))-v(\sigma(t)), x^{\Delta \Delta}(t)-v^{\Delta \Delta}(t)\right\rangle}{\|x(\sigma(t))-v(\sigma(t))\|}-M^{\Delta \Delta}(t) \quad \Delta \text {-a.e. on } A \text {. }
$$

We claim that

$$
(\|x(t)-v(t)\|-M(t))^{\Delta \Delta}>0 \quad \Delta \text {-a.e. on } A .
$$

Indeed, we deduce from the fact that $(v, M)$ is a solution-tube of (3.5) and from (3.12) that $\Delta$-almost everywhere on $\{t \in A: M(\sigma(t))>0\}$,

$$
\begin{aligned}
(\| x(t) & -v(t) \|-M(t))^{\Delta \Delta} \\
& \geq \frac{\left\langle x(\sigma(t))-v(\sigma(t)), f(t, \bar{x}(\sigma(t)))-\bar{x}(\sigma(t))+x(\sigma(t))-v^{\Delta \Delta}(t)\right\rangle}{\|x(\sigma(t))-v(\sigma(t))\|}-M^{\Delta \Delta}(t) \\
& =\frac{\left\langle\bar{x}(\sigma(t))-v(\sigma(t)), f(t, \bar{x}(\sigma(t)))-v^{\Delta \Delta}(t)\right\rangle}{M(\sigma(t))}+\|x(\sigma(t))-v(\sigma(t))\|-M(\sigma(t))-M^{\Delta \Delta}(t) \\
& >\frac{M(\sigma(t)) M^{\Delta \Delta}(t)}{M(\sigma(t))}-M^{\Delta \Delta}(t)=0,
\end{aligned}
$$

and $\Delta$-almost everywhere on $\{t \in A: M(\sigma(t))=0\}$,

$$
\begin{aligned}
(\| x(t) & -v(t) \|-M(t))^{\Delta \Delta} \\
& \geq \frac{\left\langle x(\sigma(t))-v(\sigma(t)), f(t, \bar{x}(\sigma(t)))-\bar{x}(\sigma(t))+x(\sigma(t))-v^{\Delta \Delta}(t)\right\rangle}{\|x(\sigma(t))-v(\sigma(t))\|}-M^{\Delta \Delta}(t) \\
& =\frac{\left\langle x(\sigma(t))-v(\sigma(t)), f(t, v(\sigma(t)))-v^{\Delta \Delta}(t)\right\rangle}{\|x(\sigma(t))-v(\sigma(t))\|}+\|x(\sigma(t))-v(\sigma(t))\|-M^{\Delta \Delta}(t) \\
& >-M^{\Delta \Delta}(t) \geq 0 .
\end{aligned}
$$

Observe that if $\|x(a)-v(a)\|-M(a)>0$,

$$
\|x(a)-v(a)\|^{\Delta} \geq \frac{\left\langle x(a)-v(a), x^{\Delta}(a)-v^{\Delta}(a)\right\rangle}{\|x(a)-v(a)\|} .
$$


Indeed, this follows from Proposition 2.3 when $a=\sigma(a)$. For $a<\sigma(a)$,

$$
\begin{aligned}
\|x(a)-v(a)\|^{\Delta} & =\frac{\|x(\sigma(a))-v(\sigma(a))\|-\|x(a)-v(a)\|}{\mu(a)} \\
& \geq \frac{\langle x(a)-v(a), x(\sigma(a))-v(\sigma(a))-(x(a)-v(a))\rangle}{\mu(a)\|x(a)-v(a)\|} \\
& =\frac{\left\langle x(a)-v(a), x^{\Delta}(a)-v^{\Delta}(a)\right\rangle}{\|x(a)-v(a)\|} .
\end{aligned}
$$

Similarly, if $\|x(b)-v(b)\|-M(b)>0$,

$$
\|x(\rho(b))-v(\rho(b))\|^{\Delta} \leq \frac{\left\langle x(b)-v(b), x^{\Delta}(\rho(b))-v^{\Delta}(\rho(b))\right\rangle}{\|x(b)-v(b)\|} .
$$

If (BC) denotes (3.2),

$$
\|x(a)-v(a)\|-M(a)=\|x(b)-v(b)\|-M(b) .
$$

We deduce from (3.16), (3.18), and Definition 3.1 that

$$
\|x(a)-v(a)\|-M(a)=\|x(b)-v(b)\|-M(b) \leq 0,
$$

or

$$
\begin{gathered}
\left(\|x(\rho(b))-v(\rho(b))\|^{\Delta}-M^{\Delta}(\rho(b))\right)-\left(\|x(a)-v(a)\|^{\Delta}-M^{\Delta}(a)\right) \\
\leq \leq \frac{\left\langle x^{\Delta}(\rho(b))-v^{\Delta}(\rho(b)), x(b)-v(b)\right\rangle}{\|x(b)-v(b)\|} \\
\quad-\frac{\left\langle x^{\Delta}(a)-v^{\Delta}(a), x(a)-v(a)\right\rangle}{\|x(a)-v(a)\|}-\left(M^{\Delta}(\rho(b))-M^{\Delta}(a)\right) \\
=\frac{\left\langle v^{\Delta}(a)-v^{\Delta}(\rho(b)), x(a)-v(a)\right\rangle}{\|x(a)-v(a)\|}-\left(M^{\Delta}(\rho(b))-M^{\Delta}(a)\right) \\
\leq\left\|v^{\Delta}(a)-v^{\Delta}(\rho(b))\right\|-\left(M^{\Delta}(\rho(b))-M^{\Delta}(a)\right) \leq 0 .
\end{gathered}
$$

If (BC) denotes (3.3), we deduce from (3.16), (3.18), and Definition 3.1 that

$$
\|x(a)-v(a)\|-M(a) \leq 0,
$$


or

$$
\begin{aligned}
a_{0}(\|x(a)-v(a)\|-M(a))-\gamma_{0}\left(\|x(a)-v(a)\|^{\Delta}-M^{\Delta}(a)\right) \\
\leq \frac{\left\langle x(a)-v(a), a_{0}(x(a)-v(a))-\gamma_{0}\left(x^{\Delta}(a)-v^{\Delta}(a)\right)\right\rangle}{\|x(a)-v(a)\|} \\
\quad-a_{0} M(a)+\gamma_{0} M^{\Delta}(a) \\
\leq\left\|x_{0}-\left(a_{0} v(a)-\gamma_{0} v^{\Delta}(a)\right)\right\|-a_{0} M(a)+\gamma_{0} M^{\Delta}(a) \\
\leq 0,
\end{aligned}
$$

and similarly

$$
\|x(b)-v(b)\|-M(b) \leq 0
$$

or

$$
a_{1}(\|x(b)-v(b)\|-M(b))+\gamma_{1}\left(\|x(\rho(b))-v(\rho(b))\|^{\Delta}-M^{\Delta}(\rho(b))\right) \leq 0
$$

Finally, it follows from (3.13), (3.20), (3.21), (3.22), (3.23), (3.24), (3.25), and Theorem 2.19 applied to $r(t)=\|x(t)-v(t)\|-M(t)$ that $\|x(t)-v(t)\| \leq M(t)$ for every $t \in \mathbb{T}$.

Now, we can prove the main theorem of this section.

Proof of Theorem 3.2. A solution of (3.5) is a fixed point of the operator

$$
T: C\left(\mathbb{T}, \mathbb{R}^{n}\right) \longrightarrow C\left(\mathbb{T}, \mathbb{R}^{n}\right) \text { defined by } T:=j_{0} \circ L^{-1} \circ F,
$$

where $L$ and $j_{0}$ are defined in (2.34) and Proposition 2.17, respectively. By Propositions 2.17, 2.20, and 3.3, the operator $T$ is compact. So, the Schauder fixed point theorem implies that $T$ has a fixed point and, hence, Problem (3.5) has a solution $x$. By Lemma 3.4, this solution is in $T(v, M)$. Thus, $x$ is a solution of (3.1).

In the particular case where $n=1$, as corollary of Theorem 3.2, we obtain a generalization of results of Akın [1] and Stehlík [2] for the Dirichlet and the periodic boundary conditions, respectively.

Corollary 3.5. Let $f: \mathbb{T}_{0}^{\mathcal{K}} \times \mathbb{R} \rightarrow \mathbb{R}$ be a $\Delta$-Carathéodory function. Assume that there exists $\alpha, \beta \in$ $W_{\Delta}^{2,1}(\mathbb{T}, \mathbb{R})$ such that

(i) $\alpha(t) \leq \beta(t)$ for $\Delta$-almost every $t \in \mathbb{T}$,

(ii) $\alpha^{\Delta \Delta}(t) \geq f(t, \alpha(\sigma(t)))$ and $\beta^{\Delta \Delta}(t) \leq f(t, \beta(\sigma(t)))$ for $\Delta$-almost every $t \in \mathbb{T}^{\kappa^{2}}$, 
(iii) (a) if (BC) denotes (3.2), then $\alpha(a)=\alpha(b), \alpha^{\Delta}(a) \geq \alpha^{\Delta}(\rho(b)), \beta(a)=\beta(b)$, and $\beta^{\Delta}(a) \leq \beta^{\Delta}(\rho(b))$

(b) if (BC) denotes (3.3), then $a_{0} \alpha(a)-\gamma_{0} \alpha^{\Delta}(a) \leq x_{0} \leq a_{0} \beta(a)-\gamma_{0} \beta^{\Delta}(a)$ and $a_{1} \alpha(b)+$ $\gamma_{1} \alpha^{\Delta}(\rho(b)) \leq x_{1} \leq a_{1} \beta(b)+\gamma_{1} \beta^{\Delta}(\rho(b))$,

then (3.1) has a solution $x \in W_{\Delta}^{2,1}(\mathbb{T}, \mathbb{R})$ such that $\alpha(t) \leq x(t) \leq \beta(t)$ for every $t \in \mathbb{T}$.

Proof. Observe that $((\alpha+\beta) / 2,(\beta-\alpha) / 2)$ is a solution-tube of (3.1). The conclusion follows from Theorem 3.2.

Theorem 3.2 generalizes also a result established by Henderson et al. [6] for systems of second-order dynamic equations on time scales. Let us mention that they considered a continuous map $f$ and they assumed a strict inequality in (3.27).

Corollary 3.6. Let $f: \mathbb{T}_{0}^{\kappa} \times \mathbb{R}^{n} \rightarrow \mathbb{R}^{n}$ be a $\Delta$-Carathéodory function. Assume that there exists a constant $R>0$ such that

$$
\langle x, f(t, x)\rangle \geq 0 \quad \Delta \text {-a.e. } t \in \mathbb{T}_{0}^{\kappa^{2}}, \forall x \text { such that }\|x\|=R \text {. }
$$

Moreover, if (BC) denotes (3.3), assume that $\left\|x_{0}\right\| \leq a_{0} R$ and $\left\|x_{1}\right\| \leq a_{1} R$, then the system (3.1) has a solution $x \in W_{\Delta}^{2,1}\left(\mathbb{T}, \mathbb{R}^{n}\right)$ such that $\|x(t)\| \leq R$ for every $t \in \mathbb{T}$.

Here is an example in which one cannot find a solution-tube of the form $(0, R)$.

Example 3.7. Consider the system

$$
\begin{gathered}
x^{\Delta \Delta}(t)=k(t)(\|x(\sigma(t))-\sigma(t) c\|-1) \quad \Delta \text {-a.e. } t \in \mathbb{T}_{0}^{\kappa^{2}}, \\
x(a)=0, \quad x(b)=0,
\end{gathered}
$$

where $k \in L_{\Delta}^{1}\left(\mathbb{T}_{0}, \mathbb{R}^{n} \backslash\{0\}\right)$ and $c \in \mathbb{R}^{n} \backslash\{0\}$ is such that $\|c\| \max \{|a|,|b|\} \leq 1$. One can check that $(v, M)$ is a solution-tube of (3.28) with $v(t)=t c, M(t) \equiv 1$. By Theorem 3.2, this problem has at least one solution $x$ such that $\|x(t)-t c\| \leq 1$. Observe that there is no $R>0$ such that (3.27) is satisfied.

\section{Nonlinearity Depending on $x^{\Delta}$}

In this section, we study more general systems of second-order dynamic equations on time scales. Indeed, we allow the nonlinearity $f$ to depend also on $x^{\Delta}$. We consider the problem

$$
\begin{gathered}
x^{\Delta \Delta}(t)=f\left(t, x(\sigma(t)), x^{\Delta}(t)\right), \quad \Delta \text {-a.e. } t \in \mathbb{T}_{0}^{\kappa^{2}}, \\
a_{0} x(a)-x^{\Delta}(a)=x_{0}, \quad a_{1} x(b)+\gamma_{1} x^{\Delta}(\rho(b))=x_{1},
\end{gathered}
$$

where $a_{0}, a_{1}, \gamma_{1} \geq 0$ and $\max \left\{a_{1}, \gamma_{1}\right\}>0$.

We also introduce a notion of solution-tube for this problem. 
Definition 4.1. Let $(v, M) \in W_{\Delta}^{2,1}\left(\mathbb{T}, \mathbb{R}^{n}\right) \times W_{\Delta}^{2,1}(\mathbb{T},(0, \infty))$. We say that $(v, M)$ is a solution-tube of (4.1) if

(i) for $\Delta$-almost every $t \in\left\{t \in \mathbb{T}_{0}^{\kappa^{2}}: t=\sigma(t)\right\}$,

$$
\left\langle x-v(t), f(t, x, y)-v^{\Delta \Delta}(t)\right\rangle+\left\|y-v^{\Delta}(t)\right\|^{2} \geq M(t) M^{\Delta \Delta}(t)+\left(M^{\Delta}(t)\right)^{2},
$$

for every $(x, y) \in \mathbb{R}^{2 n}$ such that $\|x-v(t)\|=M(t)$ and $\left\langle x-v(t), y-v^{\Delta}(t)\right\rangle=$ $M(t) M^{\Delta}(t)$,

(ii) for every $t \in\left\{t \in \mathbb{T}_{0}^{\kappa^{2}}: t<\sigma(t)\right\}$,

$$
\left\langle x-v(\sigma(t)), f(t, x, y)-v^{\Delta \Delta}(t)\right\rangle \geq M(\sigma(t)) M^{\Delta \Delta}(t),
$$

for every $(x, y) \in \mathbb{R}^{2 n}$ such that $\|x-v(\sigma(t))\|=M(\sigma(t))$,

(iii) $\left\|x_{0}-\left(a_{0} v(a)-v^{\Delta}(a)\right)\right\| \leq a_{0} M(a)-M^{\Delta}(a),\left\|x_{1}-\left(a_{1} v(b)+\gamma_{1} v^{\Delta}(\rho(b))\right)\right\| \leq a_{1} M(b)+$ $\gamma_{1} M^{\Delta}(\rho(b))$.

If $\mathbb{T}$ is the real interval $[a, b]$, condition (ii) of the previous definition becomes useless, and we get the notion of solution-tube introduced by the first author in [10] for a system of second-order differential equations.

Here is the main result of this section.

Theorem 4.2. Let $f: \mathbb{T}_{0}^{\kappa} \times \mathbb{R}^{2 n} \rightarrow \mathbb{R}^{n}$ be a $\Delta$-Carathéodory function. Assume that

(H1) there exists $(v, M) \in W_{\Delta}^{2,1}\left(\mathbb{T}, \mathbb{R}^{n}\right) \times W_{\Delta}^{2,1}(\mathbb{T},(0, \infty))$ a solution-tube of (4.1),

(H2) there exist constants $c, d>0$ such that $\|f(t, x, y)\| \leq c+d\|y\|$ for $\Delta$-almost every $t \in \mathbb{T}_{0}^{\kappa}$ and for every $(x, y) \in \mathbb{R}^{2 n}$ such that $\|x-v(t)\| \leq M(t)$,

then (4.1) has a solution $x \in W_{\Delta}^{2,1}\left(\mathbb{T}, \mathbb{R}^{n}\right) \cap T(v, M)$.

To prove this existence result, we consider the following modified problem:

$$
\begin{gathered}
x^{\Delta \Delta}(t)-x(\sigma(t))=g\left(t, x(\sigma(t)), x^{\Delta}(t)\right), \quad \Delta \text {-a.e. } t \in \mathbb{T}_{0}^{\kappa^{2}}, \\
a_{0} x(a)-x^{\Delta}(a)=x_{0}, \quad a_{1} x(b)+\gamma_{1} x^{\Delta}(\rho(b))=x_{1},
\end{gathered}
$$

where $g: \mathbb{T}_{0}^{\kappa} \times \mathbb{R}^{2 n} \rightarrow \mathbb{R}^{n}$ is defined by

$$
g(t, x, y)=\left\{\begin{array}{cc}
\frac{M(\sigma(t))}{\|x-v(\sigma(t))\|} f(t, \bar{x}(\sigma(t)), \tilde{y}(t))-\bar{x}(\sigma(t)) \\
+\left(1-\frac{M(\sigma(t))}{\|x-v(\sigma(t))\|}\right)\left(v^{\Delta \Delta}(t)+\frac{M^{\Delta \Delta}(t)}{\|x-v(\sigma(t))\|}(x-v(\sigma(t)))\right) \\
f(t, \bar{x}(\sigma(t)), \tilde{y}(t))-\bar{x}(\sigma(t)), & \text { if }\|x-v(\sigma(t))\|>M(\sigma(t)),
\end{array}\right.
$$


where $\bar{x}(\sigma(t))$ is defined as in (3.6),

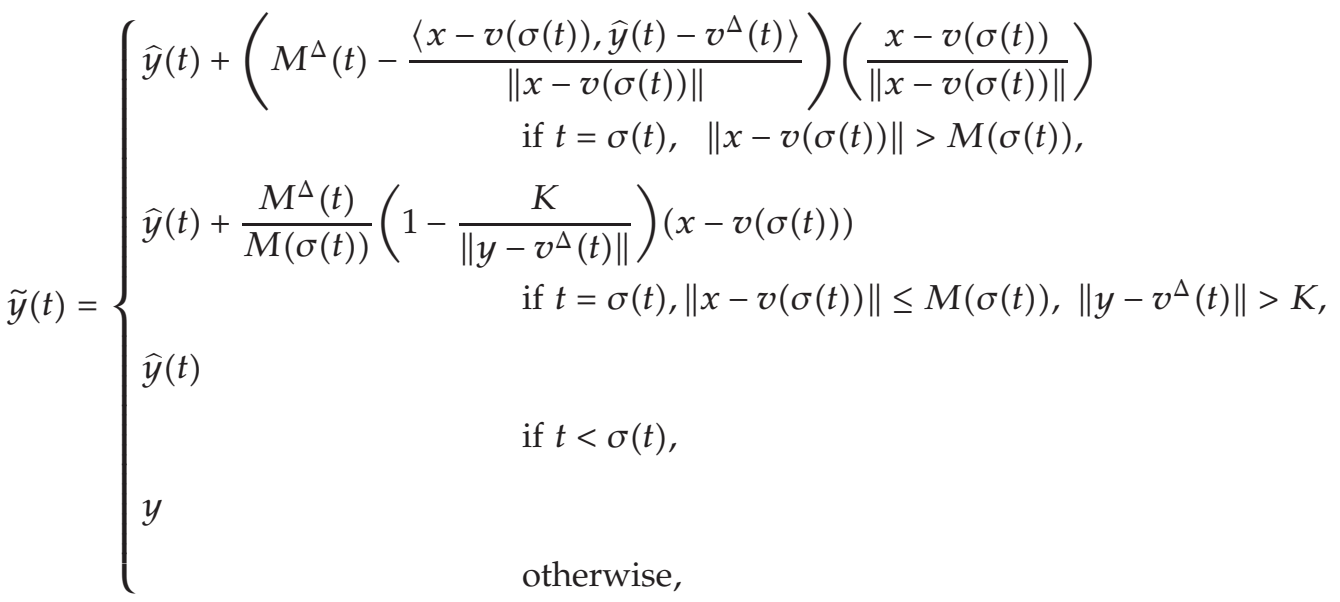

$$
\begin{aligned}
& \widehat{y}(t)= \begin{cases}\frac{K}{\left\|y-v^{\Delta}(t)\right\|}\left(y-v^{\Delta}(t)\right)+v^{\Delta}(t) & \text { if }\left\|y-v^{\Delta}(t)\right\|>K, \\
y, & \text { otherwise, }\end{cases}
\end{aligned}
$$

with $K>0$ a constant which will be fixed later.

Remark 4.3. (1) Remark that

$$
\begin{gathered}
\|\bar{x}(\sigma(t))-v(\sigma(t))\| \leq M(\sigma(t)), \\
\|\tilde{y}(t)\| \leq 2 K+\left\|v^{\Delta}(t)\right\|+\left|M^{\Delta}(t)\right| .
\end{gathered}
$$

(2) If $\|x-v(\sigma(t))\|>M(\sigma(t))$,

$$
\begin{gathered}
\|\bar{x}(\sigma(t))-v(\sigma(t))\|=M(\sigma(t)), \\
\left\langle\bar{x}(\sigma(t))-v(\sigma(t)), \widetilde{x^{\Delta}}(t)-v^{\Delta}(t)\right\rangle=M(\sigma(t)) M^{\Delta}(t), \quad \text { for } t=\sigma(t) .
\end{gathered}
$$

(3) If $\|x-v(\sigma(t))\|>M(\sigma(t))$ and $t=\sigma(t)$,

$$
\left\|\widetilde{x^{\Delta}}(t)-v^{\Delta}(t)\right\|^{2}=\left\|\widehat{x^{\Delta}}(t)-v^{\Delta}(t)\right\|^{2}+\left(M^{\Delta}(t)\right)^{2}-\frac{\left\langle x(t)-v(t), \widehat{x^{\Delta}}(t)-v^{\Delta}(t)\right\rangle^{2}}{\|x(t)-v(t)\|^{2}}
$$

(4) Since $f$ is $\Delta$-Carathéodory, by (1), there exists $h \in L_{\Delta}^{1}\left(\mathbb{T}_{0}^{\kappa}, \mathbb{R}\right)$ such that for every $x, y \in \mathbb{R}^{n}$,

$$
\|g(t, x, y)\| \leq h(t) \quad \Delta \text {-a.e. } t \in \mathbb{T}_{0}^{\kappa}
$$


We associate to $g$ the operator $G: C^{1}\left(\mathbb{T}, \mathbb{R}^{n}\right) \rightarrow L_{\Delta}^{1}\left(\mathbb{T}_{0}^{\mathcal{K}}, \mathbb{R}^{n}\right)$ defined by

$$
G(x)(t):=g\left(t, x(\sigma(t)), x^{\Delta}(t)\right)
$$

Proposition 4.4. Let $f: \mathbb{T}_{0}^{\kappa} \times \mathbb{R}^{2 n} \rightarrow \mathbb{R}^{n}$ be a $\Delta$-Carathéodory function. Assume that (H1) is satisfied, then $G$ is continuous.

Proof. Let $\left\{x_{k}\right\}$ be a sequence of $C^{1}\left(\mathbb{T}, \mathbb{R}^{n}\right)$ converging to $x \in C^{1}\left(\mathbb{T}, \mathbb{R}^{n}\right)$. It is clear that

$$
\bar{x}_{k}(t) \longrightarrow \bar{x}(t), \quad \widehat{x}_{k}(t) \longrightarrow \widehat{x^{\Delta}}(t)
$$

On $\{t \in \mathbb{T}: t<\sigma(t)\}$, we have

$$
g\left(t, x_{k}(\sigma(t)), x_{k}^{\Delta}(t)\right) \longrightarrow g\left(t, x(\sigma(t)), x^{\Delta}(t)\right)
$$

since $f$ is $\Delta$-Carathéodory.

Similarly, $\Delta$-almost everywhere on $\left\{t \in \mathbb{T}_{0}^{\kappa}: t=\sigma(t),\|x(\sigma(t))-v(\sigma(t))\| \neq M(\sigma(t))\right\}$,

$$
g\left(t, x_{k}(\sigma(t)), x_{k}^{\Delta}(t)\right) \longrightarrow g\left(t, x(\sigma(t)), x^{\Delta}(t)\right)
$$

since ${\widetilde{x^{\Delta}}}_{k}(t) \rightarrow \widetilde{x^{\Delta}}(t)$ and, for $k$ sufficiently large, $\left\|x_{k}(\sigma(t))-v(\sigma(t))\right\| \neq M(\sigma(t))$.

Denote $S:=\left\{t \in \mathbb{T}_{0}^{\mathcal{K}}: t=\sigma(t)\right.$ and $\left.\|x(\sigma(t))-v(\sigma(t))\|=M(\sigma(t))\right\}$ and $I_{t}=\{k \in \mathbb{N}$ : $\left.\left\|x_{k}(\sigma(t))-v(\sigma(t))\right\| \leq M(\sigma(t))\right\}$. As before, it is easy to check that $\Delta$-almost everywhere on $\left\{t \in S:\right.$ card $\left.I_{t}=\infty\right\}$,

$$
g\left(t, x_{k}(\sigma(t)), x_{k}^{\Delta}(t)\right) \longrightarrow g\left(t, x(\sigma(t)), x^{\Delta}(t)\right) \text { as } k \in I_{t} \text { goes to infinity. }
$$

On the other hand, Proposition 2.11 implies that

$$
\left\langle x(\sigma(t))-v(\sigma(t)), x^{\Delta}(t)-v^{\Delta}(t)\right\rangle=M(\sigma(t)) M^{\Delta}(t) \quad \Delta \text {-a.e. } t \in S \text {. }
$$


So, $\Delta$-almost everywhere on $\left\{t \in S: \operatorname{card}\left(\mathbb{N} \backslash I_{t}\right)=\infty\right\}$,

$$
\begin{aligned}
\widetilde{x_{k}^{\Delta}}(t)= & \widehat{x_{k}^{\Delta}}(t) \\
& +\left(M^{\Delta}(t)-\frac{\left\langle x_{k}(\sigma(t))-v(\sigma(t)), \widehat{x_{k}^{\Delta}}(t)-v^{\Delta}(t)\right\rangle}{\left\|x_{k}(\sigma(t))-v(\sigma(t))\right\|}\right)\left(\frac{x_{k}(\sigma(t))-v(\sigma(t))}{\left\|x_{k}(\sigma(t))-v(\sigma(t))\right\|}\right) \\
\longrightarrow & \widehat{x^{\Delta}}(t) \\
& +\left(M^{\Delta}(t)-\frac{\left\langle x(\sigma(t))-v(\sigma(t)), \widehat{x^{\Delta}}(t)-v^{\Delta}(t)\right\rangle}{\|x(\sigma(t))-v(\sigma(t))\|}\right)\left(\frac{x(\sigma(t))-v(\sigma(t))}{\|x(\sigma(t))-v(\sigma(t))\|}\right) \\
= & \left\{\begin{array}{rr}
\widehat{x^{\Delta}}(t)+\frac{M^{\Delta}(t)}{M(\sigma(t))}\left(1-\frac{K}{\left\|x^{\Delta}(t)-v^{\Delta}(t)\right\|}\right)(x(\sigma(t))-v(\sigma(t))) \\
\widehat{x^{\Delta}}(t) & \text { if }\left\|x^{\Delta}(t)-v^{\Delta}(t)\right\|>K, \\
= & \text { if }\left\|x^{\Delta}(t)-v^{\Delta}(t)\right\| \leq K,
\end{array}\right.
\end{aligned}
$$

as $k \in \mathbb{N} \backslash I_{t}$ goes to infinity. Thus, $\Delta$-almost everywhere on $\left\{t \in S: \operatorname{card}\left(\mathbb{N} \backslash I_{t}\right)=\infty\right\}$,

$g\left(t, x_{k}(\sigma(t)), x_{k}^{\Delta}(t)\right) \longrightarrow g\left(t, x(\sigma(t)), x^{\Delta}(t)\right)$ as $k \in \mathbb{N} \backslash I_{t}$ goes to infinity.

By Remark 4.3(4), we have

$$
\left\|g\left(t, x_{k}(\sigma(t)), x_{k}^{\Delta}(t)\right)\right\| \leq h(t) \quad \Delta \text {-a.e. } t \in \mathbb{T}_{0}^{\kappa}
$$

Theorem 2.8 implies that

$$
G\left(x_{k}\right) \longrightarrow G(x) \quad \text { in } L_{\Delta}^{1}\left(\mathbb{T}_{0}^{\kappa}, \mathbb{R}^{n}\right)
$$

Lemma 4.5. Assume (H1), then $x \in T(v, M)$ for every solution $x$ of (4.4).

Proof. Observe that $x^{\Delta \Delta}(t)$ (resp., $v^{\Delta \Delta}(t), M^{\Delta \Delta}(t)$ ) exists $\Delta$-almost everywhere on $\mathbb{T}^{\kappa^{2}}$, since $x \in W_{\Delta}^{2,1}\left(\mathbb{T}, \mathbb{R}^{n}\right)\left(\right.$ resp., $\left.v \in W_{\Delta}^{2,1}\left(\mathbb{T}, \mathbb{R}^{n}\right), M \in W_{\Delta}^{2,1}(\mathbb{T}, \mathbb{R})\right)$. Denote

$$
A=\left\{t \in \mathbb{T}_{0}^{\kappa^{2}}:\|x(\sigma(t))-v(\sigma(t))\|>M(\sigma(t))\right\} .
$$


Observe that by (H1) and Remark 4.3(2), for $\Delta$-almost every $t \in A$,

$$
\begin{aligned}
&\left\langle x(\sigma(t))-v(\sigma(t)), x^{\Delta \Delta}(t)-v^{\Delta \Delta}(t)\right\rangle \\
&=\left\langle x(\sigma(t))-v(\sigma(t)), g\left(t, x(\sigma(t)), x^{\Delta}(t)\right)+x(\sigma(t))-v^{\Delta \Delta}(t)\right\rangle \\
&=\left\langle\bar{x}(\sigma(t))-v(\sigma(t)), f\left(t, \bar{x}(\sigma(t)), \widetilde{x^{\Delta}}(t)\right)-v^{\Delta \Delta}(t)\right\rangle \\
&+M^{\Delta \Delta}(t)(\|x(\sigma(t))-v(\sigma(t))\|-M(\sigma(t))) \\
&+\|x(\sigma(t))-v(\sigma(t))\|-M(\sigma(t)) \\
&>\left\langle\bar{x}(\sigma(t))-v(\sigma(t)), f\left(t, \bar{x}(\sigma(t)), \widetilde{x^{\Delta}}(t)\right)-v^{\Delta \Delta}(t)\right\rangle \\
&+M^{\Delta \Delta}(t)(\|x(\sigma(t))-v(\sigma(t))\|-M(\sigma(t))) \\
& \geq \begin{cases}M^{\Delta \Delta}(t)\|x(\sigma(t))-v(\sigma(t))\| \\
M^{\Delta \Delta}(t)\|x(\sigma(t))-v(\sigma(t))\|+\left(M^{\Delta}(t)\right)^{2}-\left\|\widetilde{x^{\Delta}}(t)-v^{\Delta}(t)\right\|^{2} & \text { if } t=\sigma(t) .\end{cases}
\end{aligned}
$$

This inequality with Lemma 2.4(1) imply that for $\Delta$-almost every $t \in\{t \in A: t<\sigma(t)\}$,

$$
(\|x(t)-v(t)\|-M(t))^{\Delta \Delta}>0 .
$$

Also, (4.22), Lemma 2.4(2), and Remark 4.3(3) imply that for $\Delta$-almost every $t \in\{t \in A: t=$ $\sigma(t)\}$,

$$
\begin{aligned}
& (\|x(t)-v(t)\|-M(t))^{\Delta \Delta} \\
& >\frac{\left(M^{\Delta}(t)\right)^{2}-\left\|\widetilde{x^{\Delta}}(t)-v^{\Delta}(t)\right\|^{2}+\left\|x^{\Delta}(t)-v^{\Delta}(t)\right\|^{2}}{\|x(t)-v(t)\|} \\
& -\frac{\left\langle x(t)-v(t), x^{\Delta}(t)-v^{\Delta}(t)\right\rangle^{2}}{\|x(t)-v(t)\|^{3}} \\
& =\frac{\left\|x^{\Delta}(t)-v^{\Delta}(t)\right\|^{2}-\left\|\widehat{x^{\Delta}}(t)-v^{\Delta}(t)\right\|^{2}}{\|x(t)-v(t)\|} \\
& +\frac{\left\langle x(t)-v(t), \widehat{x^{\Delta}}(t)-v^{\Delta}(t)\right\rangle^{2}-\left\langle x(t)-v(t), x^{\Delta}(t)-v^{\Delta}(t)\right\rangle^{2}}{\|x(t)-v(t)\|^{3}} \\
& =\left\{\begin{array}{c}
0 \\
\text { if }\left\|x^{\Delta}(t)-v^{\Delta}(t)\right\| \leq K, \\
\left(1-\frac{K^{2}}{\left\|x^{\Delta}(t)-v^{\Delta}(t)\right\|^{2}}\right)\left(\frac{\left\|x^{\Delta}(t)-v^{\Delta}(t)\right\|^{2}}{\|x(t)-v(t)\|}-\frac{\left\langle x(t)-v(t), x^{\Delta}(t)-v^{\Delta}(t)\right\rangle^{2}}{\|x(t)-v(t)\|^{3}}\right) \\
\text { if }\left\|x^{\Delta}(t)-v^{\Delta}(t)\right\|>K,
\end{array}\right.
\end{aligned}
$$

$\geq 0$. 
Let us denote $r(t)=\|x(t)-v(t)\|-M(t)$. Inequalities (4.23) and (4.24) imply that $r^{\Delta \Delta}(t)>0$ for $\Delta$-almost every $t \in\left\{t \in \mathbb{T}_{0}^{\kappa^{2}}: r(\sigma(t))>0\right\}$. Arguing as in the proof of Lemma 3.4, we can show that

$$
\begin{gathered}
r(a) \leq 0 \quad \text { or } \quad a_{0} r(a)-r^{\Delta}(a) \leq 0, \\
r(b) \leq 0 \quad \text { or } \quad a_{1} r(b)+\gamma_{1} r^{\Delta}(\rho(b)) \leq 0 .
\end{gathered}
$$

Theorem 2.19 implies that $x \in T(v, M)$.

We can now prove the existence theorem of this section.

Proof of Theorem 4.2. We first show that for every solution $x$ of (4.4), there exists a constant $K>0$ such that

$$
\left\|x^{\Delta}(t)-v^{\Delta}(t)\right\| \leq K \quad \text { for every } t \in \mathbb{T}^{\kappa}
$$

By (H2), Proposition 2.13 and Lemma 4.5, for any $x$ solution of (4.4), we have for $\Delta$-almost every $t \in \mathbb{T}^{\kappa}$,

$$
\begin{aligned}
\left\|x^{\Delta}(t)\right\| & \leq\left\|x^{\Delta}(a)\right\|+\int_{[a, t) \cap \mathbb{T}}\left\|x^{\Delta \Delta}(s)\right\| \Delta s \\
& =\left\|x_{0}-a_{0} x(a)\right\|+\int_{[a, t) \cap \mathbb{T}}\left\|f\left(s, x(\sigma(s)), \widetilde{x^{\Delta}}(s)\right)\right\| \Delta s \\
& \leq c_{0}+\int_{[a, t) \cap \mathbb{T}} c+d\left\|\widetilde{x^{\Delta}}(s)\right\| \Delta s \\
& \leq c_{0}+\int_{[a, t) \cap \mathbb{T}} c+d\left(\left\|\widehat{x^{\Delta}}(s)-v^{\Delta}(s)\right\|+\left\|v^{\Delta}(s)\right\|+\left|M^{\Delta}(s)\right|\right) \Delta s \\
& \leq c_{0}+\int_{[a, t) \cap \mathbb{T}} c+d\left(\left\|x^{\Delta}(s)-v^{\Delta}(s)\right\|+\left\|v^{\Delta}(s)\right\|+\left|M^{\Delta}(s)\right|\right) \Delta s \\
& \leq c_{1}+\int_{[a, t) \cap \mathbb{T}} d\left\|x^{\Delta}(s)\right\| \Delta s,
\end{aligned}
$$

where

$$
\begin{aligned}
& c_{0}:=\left\|x_{0}\right\|+a_{0}(M(a)+\|v(a)\|) \\
& c_{1}:=c_{0}+\int_{[a, b) \cap \mathbb{T}} c+d\left(2\left\|v^{\Delta}(s)\right\|+\left|M^{\Delta}(s)\right|\right) \Delta s .
\end{aligned}
$$

Fix $K \geq\left\|v^{\Delta}\right\|_{0}+c_{1}\left\|e_{d}(\cdot, a)\right\|_{0}$. By Theorem 2.5,

$$
\left\|x^{\Delta}(t)-v^{\Delta}(t)\right\| \leq\left\|x^{\Delta}(t)\right\|+\left\|v^{\Delta}(t)\right\| \leq\left\|v^{\Delta}(t)\right\|+c_{1} e_{d}(t, a) \leq K \quad \forall t \in \mathbb{T}^{\kappa} .
$$


Consider the operator

$$
T=j_{1} \circ L^{-1} \circ G: C^{1}\left(\mathbb{T}, \mathbb{R}^{n}\right) \longrightarrow C^{1}\left(\mathbb{T}, \mathbb{R}^{n}\right),
$$

where $L$ and $j_{1}$ are defined, respectively, in (2.34) and Proposition 2.16. By Propositions 2.16, 2.20, and 4.4, $T$ is continuous. Moreover, $T$ is compact. Indeed, by Remark 4.3(4), there exists $h \in L_{\Delta}^{1}\left(\mathbb{T}_{0}^{\kappa},[0, \infty)\right)$ such that for every $z \in T\left(C^{1}\left(\mathbb{T}, \mathbb{R}^{n}\right)\right)$, there exists $x \in C^{1}\left(\mathbb{T}, \mathbb{R}^{n}\right)$ such that $z=T(x)$ and

$$
\|G(x)(s)\| \leq h(s) \quad \Delta \text {-a.e. } t \in \mathbb{T}_{0}^{\kappa} .
$$

Since $j_{1}$ and $L^{-1}$ are continuous and affine, they map bounded sets in bounded sets. Thus, there exists a constant $k_{0}>0$ such that

$$
\|z\|_{1} \leq k_{0}
$$

Moreover, $z \in W_{\Delta}^{2,1}\left(\mathbb{T}, \mathbb{R}^{n}\right)$ and

$$
L(z)(s)=z^{\Delta \Delta}(s)-z(\sigma(s))=G(x)(s) \quad \Delta \text {-a.e. } s \in \mathbb{T}^{\kappa^{2}} .
$$

So, for every $t<\tau$ in $\mathbb{T}^{\kappa}$,

$$
\left\|z^{\Delta}(t)-z^{\Delta}(\tau)\right\| \leq \int_{[t, \tau) \cap \mathbb{T}}\|z(\sigma(s))+G(x)(s)\| \Delta s \leq \int_{[t, \tau) \cap \mathbb{T}} k_{0}+h(s) \Delta s .
$$

Thus, $T\left(C^{1}\left(\mathbb{T}, \mathbb{R}^{n}\right)\right)$ is bounded and equicontinuous in $C^{1}\left(\mathbb{T}, \mathbb{R}^{n}\right)$. By an analog of the ArzelàAscoli Theorem for our context, $T\left(C^{1}\left(\mathbb{T}, \mathbb{R}^{n}\right)\right)$ is relatively compact in $C^{1}\left(\mathbb{T}, \mathbb{R}^{n}\right)$.

By the Schauder fixed point theorem, $T$ has a fixed point $x$ which is a solution of (4.4). By Lemma 4.5, $x \in T(v, M)$. Also, $x$ satisfies (4.26). Hence, $x$ is also a solution of (4.1).

Here is an example in which one cannot find a solution-tube of the form $(0, R)$. Moreover, Assumption (A2) stated in the introduction and assumed in [9] is not satisfied.

Example 4.6. Let $\mathbb{T}=[0,1] \cup\{2\} \cup[3,4]$ and consider the system

$$
\begin{gathered}
x^{\Delta \Delta}(t)=x(\sigma(t))\left(l(t)+\left\|x^{\Delta}(t)\right\|\right)+k(t) \quad \Delta \text {-a.e. } t \in \mathbb{T}_{0}, \\
x^{\Delta}(0)=x_{0}, \quad x^{\Delta}(4)=x_{1}
\end{gathered}
$$

where $x_{0}, x_{1} \in \mathbb{R}^{n}$ are such that $\left\|x_{0}\right\| \leq 1,\left\|x_{1}\right\| \leq 1, l \in L_{\Delta}^{1}\left(\mathbb{T}_{0},[0, \infty)\right)$, and $k \in L_{\Delta}^{1}\left(\mathbb{T}_{0}, \mathbb{R}^{n}\right)$ such that $l(1) \geq 1, l(2) \geq 1,\|k(t)\| \leq 2$, and $l(t) \leq r$ for $\Delta$-almost every $t \in \mathbb{T}_{0}$ for some $r>0$. 
Take $v(t) \equiv 0$ and

$$
M(t)= \begin{cases}5-t & \text { if } t \in[0,2] \cap \mathbb{T} \\ t & \text { if } t \in[3,4] \cap \mathbb{T} .\end{cases}
$$

So, $v \in W_{\Delta}^{2,1}\left(\mathbb{T}, \mathbb{R}^{n}\right), M \in W_{\Delta}^{2,1}(\mathbb{T},(0, \infty))$, and

$$
\begin{aligned}
& M^{\Delta}(t)= \begin{cases}-1 & \text { if } t \in[0,1] \\
0 & \text { if } t=2, \\
1 & \text { if } t \in[3,4]\end{cases} \\
& M^{\Delta \Delta}(t)= \begin{cases}0 & \text { if } t \in[0,1), \\
1 & \text { if } t \in\{1,2\} \\
0 & \text { if } t \in[3,4]\end{cases}
\end{aligned}
$$

One has $\left\|x_{0}\right\| \leq-M^{\Delta}(0)$ and $\left\|x_{1}\right\| \leq M^{\Delta}(4)$. Observe that $\Delta$-almost everywhere on $[0,1) \cup$ $[3,4]$ if $\|x\|=M(t)$ and $\langle x, y\rangle=M(t) M^{\Delta}(t)$, one has $\|y\| \geq 1$ and

$$
\begin{aligned}
\langle x, f(t, x, y)\rangle+\|y\|^{2} & =\|x\|^{2}(l(t)+\|y\|)+\langle x, k(t)\rangle+\|y\|^{2} \\
& \geq M(t)(M(t)-\|k(t)\|)+1 \\
& \geq 1 \\
& =M(t) M^{\Delta \Delta}(t)+\left(M^{\Delta}(t)\right)^{2} .
\end{aligned}
$$

If $t \in\{1,2\}$ and $\|x\|=M(\sigma(t))=3$,

$$
\begin{aligned}
\langle x, f(t, x, y)\rangle & =\|x\|^{2}(l(t)+\|y\|)+\langle x, k(t)\rangle \\
& \geq 9 l(t)-3\|k(t)\| \\
& \geq 3 \\
& =M(\sigma(t)) M^{\Delta \Delta}(t) .
\end{aligned}
$$

So, $(v, M)$ is a solution-tube of (4.35). Moreover,

$$
\|f(t, x, y)\| \leq 2+5 r+5\|y\| \quad \Delta \text {-a.e. } t \in \mathbb{T}_{0} \text {, all }(x, y) \text { such that }\|x\| \leq M(t) .
$$

Theorem 4.2 implies that (4.35) has at least one solution $x$ such that $\|x(t)\| \leq M(t)$. Observe that if $x_{0} \neq 0$ or $x_{1} \neq 0$, this problem has no solution-tube of the form $(0, R)$ with $R$ a positive constant since Definition 4.1(iii) would not be satisfied. This explains why Henderson and 
Tisdell [9] did not consider the Neumann boundary condition. Notice also that the restriction $d(\rho(b)-a)$ in $(\mathrm{A} 2)$ is not satisfied in this example.

\section{Acknowledgments}

The authors would like to thank, respectively, CRSNG-Canada and FQRNT-Québec for their financial support.

\section{References}

[1] E. Akın, "Boundary value problems for a differential equation on a measure chain," Panamerican Mathematical Journal, vol. 10, no. 3, pp. 17-30, 2000.

[2] P. Stehlík, "Periodic boundary value problems on time scales," Advances in Difference Equations, no. 1, pp. 81-92, 2005.

[3] A. C. Peterson, Y. N. Raffoul, and C. C. Tisdell, "Three point boundary value problems on time scales," Journal of Difference Equations and Applications, vol. 10, no. 9, pp. 843-849, 2004.

[4] P. Stehlík, "On lower and upper solutions without ordering on time scales," Advances in Difference Equations, Article ID 73860, 12 pages, 2006.

[5] C. C. Tisdell and H. B. Thompson, "On the existence of solutions to boundary value problems on time scales," Dynamics of Continuous, Discrete E Impulsive Systems. Series A. Mathematical Analysis, vol. 12, no. 5, pp. 595-606, 2005.

[6] J. Henderson, A. Peterson, and C. C. Tisdell, "On the existence and uniqueness of solutions to boundary value problems on time scales," Advances in Difference Equations, no. 2, pp. 93-109, 2004.

[7] P. Amster, C. Rogers, and C. C. Tisdell, "Existence of solutions to boundary value problems for dynamic systems on time scales," Journal of Mathematical Analysis and Applications, vol. 308, no. 2, pp. 565-577, 2005.

[8] F. M. Atici, A. Cabada, C. J. Chyan, and B. Kaymakçalan, "Nagumo type existence results for secondorder nonlinear dynamic BVPs," Nonlinear Analysis. Theory, Methods $\mathcal{E}$ Applications, vol. 60, no. 2, pp. 209-220, 2005.

[9] J. Henderson and C. C. Tisdell, "Dynamic boundary value problems of the second-order: BernsteinNagumo conditions and solvability," Nonlinear Analysis. Theory, Methods E Applications, vol. 67, no. 5, pp. 1374-1386, 2007.

[10] M. Frigon, "Boundary and periodic value problems for systems of nonlinear second order differential equations," Topological Methods in Nonlinear Analysis, vol. 1, no. 2, pp. 259-274, 1993.

[11] J. Zhou and Y. Li, "Sobolev's spaces on time scales and its applications to a class of second order Hamiltonian systems on time scales," Nonlinear Analysis. Theory, Methods E Applications, vol. 73, no. 5, pp. 1375-1388, 2010.

[12] M. Bohner and G. Guseinov, "Riemann and Lebesgue integration," in Advances in Dynamic Equations on Time Scales, pp. 117-163, Birkhäuser, Boston, Mass, USA, 2003.

[13] M. Bohner and A. Peterson, Dynamic Equations on Time Scales: An Introduction with Application, Birkhäuser, Boston, Mass, USA, 2001.

[14] A. Cabada and D. R. Vivero, "Expression of the Lebesgue $\Delta$-integral on time scales as a usual Lebesgue integral: application to the calculus of $\Delta$-antiderivatives," Mathematical and Computer Modelling, vol. 43, no. 1-2, pp. 194-207, 2006.

[15] S. Hilger, "Analysis on measure chains - a unified approach to continuous and discrete calculus," Results in Mathematics, vol. 18, no. 1-2, pp. 18-56, 1990.

[16] A. Cabada and D. R. Vivero, "Criterions for absolute continuity on time scales," Journal of Difference Equations and Applications, vol. 11, no. 11, pp. 1013-1028, 2005.

[17] H. Gilbert, "Existence Theorems for First-Order Equations on Time Scales with $\Delta$-Carathéodory Functions," Advances in Difference Equations, vol. 2010, Article ID 650827, 20 pages, 2010.

[18] R. P. Agarwal, V. Otero-Espinar, K. Perera, and D. R. Vivero, “Basic properties of Sobolev's spaces on time scales," Advances in Difference Equations, Article ID 38121, 14 pages, 2006.

[19] G. T. Bhaskar, "Comparison theorem for a nonlinear boundary value problem on time scales. Dynamic equations on time scale," Journal of Computational and Applied Mathematics, vol. 141, no. 1-2, pp. 117122, 2002. 


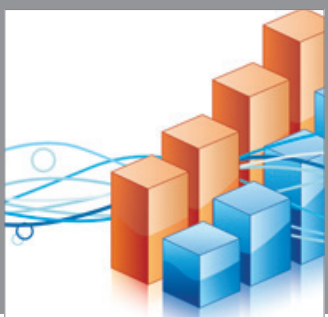

Advances in

Operations Research

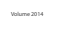

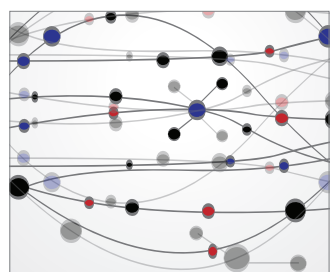

\section{The Scientific} World Journal
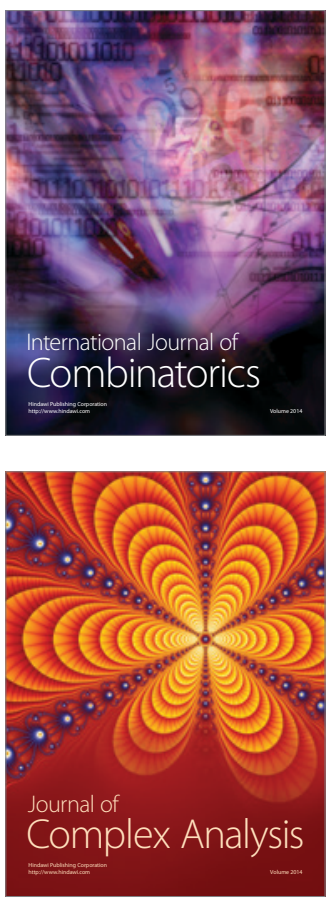

International Journal of

Mathematics and

Mathematical

Sciences
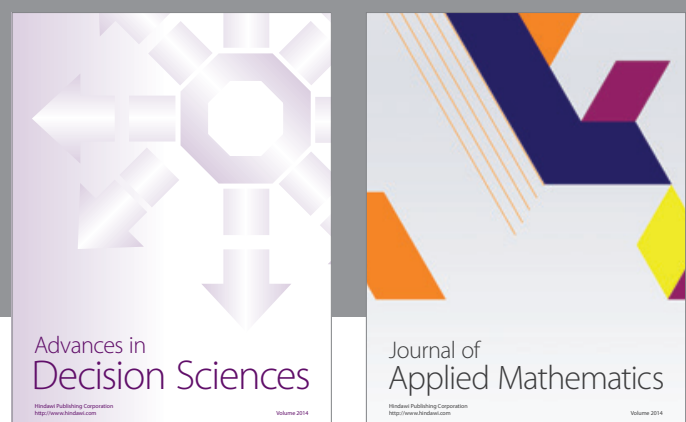

Journal of

Applied Mathematics
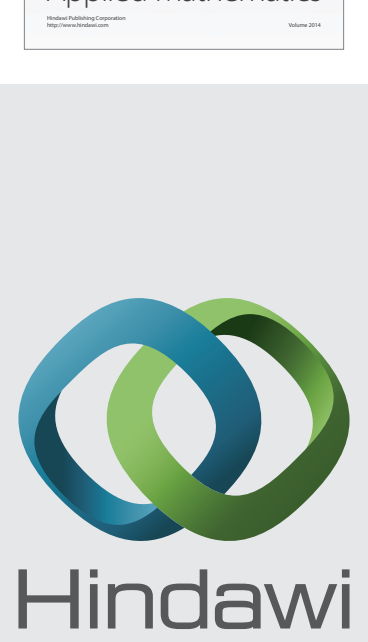

Submit your manuscripts at http://www.hindawi.com
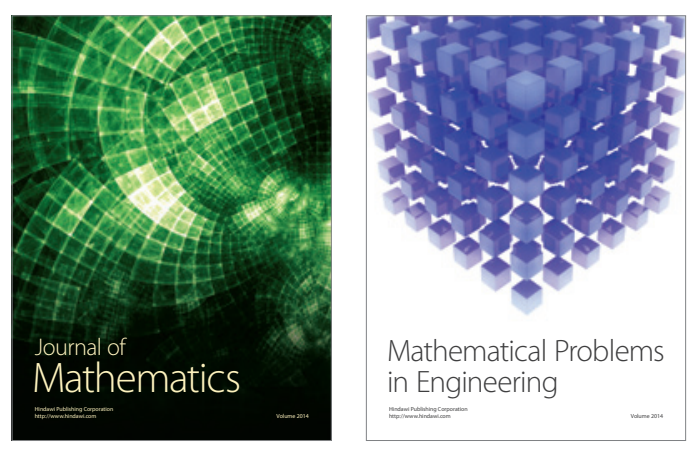

Mathematical Problems in Engineering
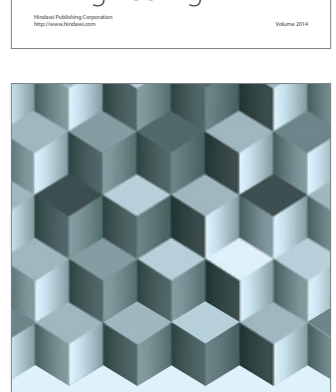

Journal of

Function Spaces
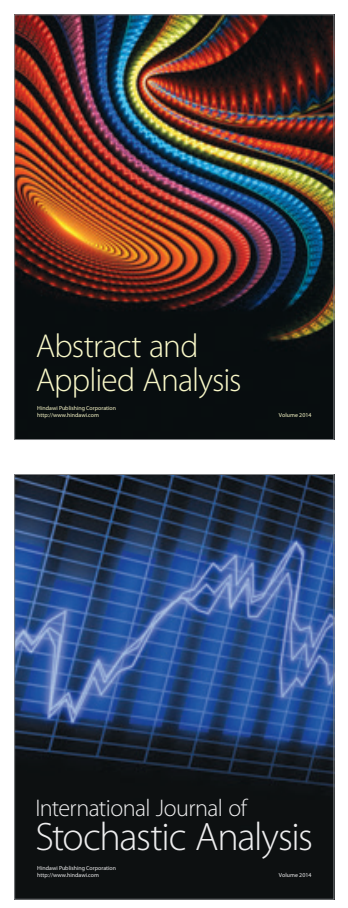

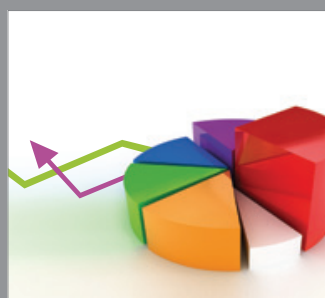

ournal of

Probability and Statistics

Promensencen
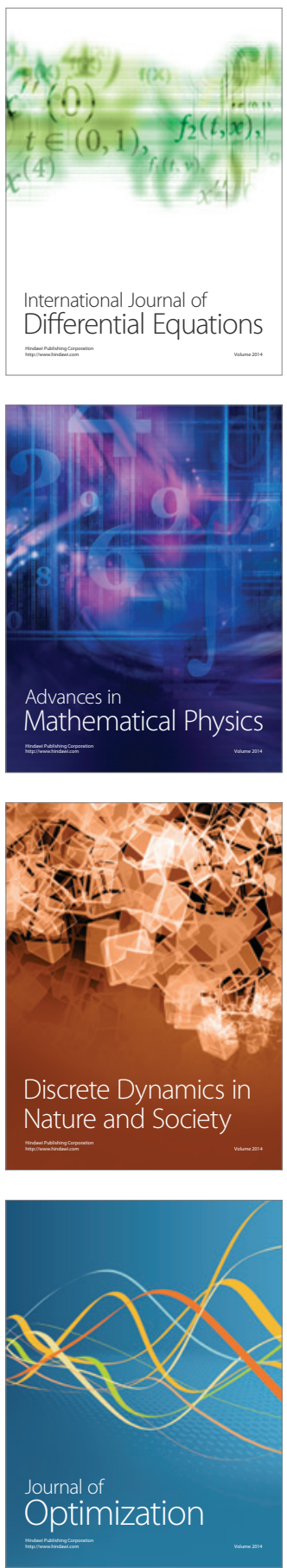